\title{
The response of ice cover to a load moving along a frozen channel
}

\author{
Konstantin Shishmareva,*, Tatyana Khabakhpasheva ${ }^{\mathrm{b}, \mathrm{c}}$, Alexander Korobkin ${ }^{\mathrm{c}}$ \\ ${ }^{a}$ Altai State University, Barnaul, Russia \\ ${ }^{b}$ Lavrentyev Institute of Hydrodynamics, Novosibirsk, Russia \\ ${ }^{c}$ School of Mathematics, University of East Anglia, Norwich, UK
}

\begin{abstract}
Deflections and stresses in an ice cover of a frozen channel caused by a load moving with a constant speed along the channel are studied. The channel is of rectangular cross section. The ice cover is isotropic and clamped to the walls of the channel. The fluid in the channel is inviscid and incompressible. The external load is modeled by a localized smooth pressure distribution moving along the central line of the channel. The ice cover is modeled as a viscoelastic plate. Deflection of the ice and strains in the ice plate are independent of time in the coordinate system moving together with the load. The effect of the channel walls on the ice response is studied. This effect can be significant in experiments with loads moving in ice tanks. The linear hydroelastic problem is solved by using the Fourier transform along the channel and the method of normal modes across the channel. It is found that the presence of the vertical walls of the channel reduces the ice deflection but increases the elastic strains in the ice plate. The effects of the load speed, width and depth of the channel on the hydroelastic response of the ice cover are studied in detail. In contrast to the problem of a load moving on ice sheets of infinite extent, there are infinitely many critical speeds of hydroelastic waves in a frozen channel. Correspondingly, there are many values of the speeds of a moving load at which the stresses in the ice cover are amplified. The obtained deflections and strains in the canned ice cover are compared with the corresponding solutions for the infinite ice plate and with the solutions of simplified problems without account for the dynamic component of the liquid pressure. It is shown that the models of ice response without hydrodynamic component of the pressure provide correct stresses in the ice sheet only for very low speeds of the moving load.
\end{abstract}

Keywords: Viscoelastic plate, Moving loads, Ice defections and strains

\section{Introduction}

The problem of ice deflection caused by a moving load was studied in the past for an infinite ice plate (see [1] for an excellent review of available results and approaches). The load moving at a constant speed along a straight line was modelled by a point pressure or a smooth and localised distribution of external 5 pressure. The problem was investigated within the linear theory of hydroelasticity (see [1] [2] [3] [4] [5]) and fully nonlinear model (see [6] [7] [8] [9] [10]). Transient problems of moving loads with application to the aircraft landing on ice, in particular, were studied in [11] [12] [13] [14] [15]. The problem of loads moving

\footnotetext{
* Corresponding author

Email address: shishmarev.k@mail.ru (Konstantin Shishmarev)
} 
along a channel covered with ice received less attention. This problem is important because laboratory experiments with moving loads are performed in ice tanks of finite width. Newman in his recent paper "Channel wall effects in radiation-diffraction analysis" [16] wrote "Computations of wave-body interactions are usually performed for an unbounded horizontal domain, whereas experiments are performed in tanks of finite width. Reflections from the tank walls can be significant if the width is of the same order as the body length or wavelength, especially when the forward speed of the body is small or zero." This statement is applicable to ice channels as well, as it is shown in the present paper.

The problem is also practically important for narrow water ways, such as rivers and channels, frozen bays and straits [17] Frozen rivers in northern regions can be used for transportation in winter time. We need to be sure that the ice on the river is strong enough to support a certain weight of the cargo transported at a certain speed along the ice. On the other hand, in some situations, as, for example, flooding in early spring on some northern rivers, the ice on the river should be broken and removed. In particular, severe flooding has happened on the Lena River in 2007. In early spring headwaters of the river were free from the ice, while lower part of the river was still covered with ice. This resulted in the water accumulation and the formation of a temporary water reservoir. According to the Dartmouth Flood Observatory [18]: "the May floods along the Lena and its tributaries inundated more than 1,000 houses, put 12 towns under water, damaged or destroyed 41 bridges, and affected more than 14,000 people". Another example is the flooding on the Yellow River in China in 2014 [19]. To prevent the flooding, remote frozen parts of the river were bombarded by Chinese Air Force. Twenty four bombs were dropped on the frozen river in order to free up the flow and save towns and cities upstream from flooding. The ice cover has to be broken also between two hydroelectric dams build in cascade to prevent high loads on the dam downstream due to a dam-break wave coming from upstream [20]. The dam-break wave propagates towards the edge of the ice between two dams as a free-surface wave. Then the wave is divided into two waves: a pressure wave propagating under the ice cover and a free-surface wave propagating over the cover. The resulting loads propagate further and may produce high hydrodynamic pressures on the downstream dam leading to its failure.

To break ice covers in both rivers and offshore, air-cushion vehicles or hovercraft can be used. The vehicle moves along the ice cover at a certain speed generating stresses in it which are large enough to break the ice (see [17], [21], [22], [23] and [25]). It was shown that air-cushion vehicles can make very effective icebreakers. It is written in [21]: "The measurements of sheet deflections in the Memorial University of Newfoundland wave tank and the Institute for Marine Dynamics ice tank showed that a critical speed exists for motion over a sheet. At this speed, sheet deflections are limited only by dissipation and nonlinearities. We believe this critical speed is the source of high speed mode hovercraft icebreaking." Squire in [1], p.200 wrote about the critical speed of hydroelastic waves: "Phase speed $c$ has minimum, denoted by $c_{m i n}$, above which flexural-gravity waves can propagate freely and below which no such waves are generated. The minimum is associated with the critical speed $v_{\text {crit }}$ at which deflection of the floating ice plate is greatest when a load travels by." The corresponding method of icebreaking was studied both theoretically, numerically [23, 25] and experimentally [17] by Kozin and his group. In this method, so-called as "resonant method of icebreaking", air-cushion vehicle moves at a speed close to the critical speed of hydroelastic waves in the ice sheet. 
Depending on environmental conditions and place on a river or offshore, where the ice have to be broken and removed, the "resonant method of icebreaking" is supplemented with some other techniques to increase the stresses in the ice. It is written in [25]: "traditional tools and technologies (icebreakers, icebreaking consoles, explosions, explosives charges, etc.) used to solve ice breaking problems often do not lead to appropriate results. Icebreakers and compounds with icebreaking prefixes are not able to destroy the ice cover on shallow water, they are not effective in destructing ice jams and ice hanging dams. One of the ways to avoid these disadvantages is to use the resonance method, which reduces energy costs compared with existing methods." The success and effectiveness of this method depend on how well the ice response can be predicted for realistic conditions, including the presence of the ice boundaries. In [17], page 23, it is reported that there is an optimum distance of the air-cushion vehicle trajectory from the sea coast, at which less energy is required to break the ice. Several experimental campaigns and sea trials are described in [17], however, not many results are reported in this book. The experiments were performed in ice tanks (see [17], pages 147-155) aiming to investigate the effect of the tank vertical walls on the breaking capacity of a load moving along the artificial ice in the tank. The experimental results were compared with numerical predictions in [25]. The water depth was $10 \mathrm{~cm}$, critical load speed was reported as $1 \mathrm{~m} / \mathrm{s}$ and the distance between the walls of the tank was varied from 20 to $70 \mathrm{~cm}$. Dependence of the critical speed on the width of the channel was not taken into account. It was reported that the numerical results by a finite-element method are similar to the experimental results qualitatively but differ from them in magnitude due to the edge conditions at the walls. The elastic plate was loosely clamped to the walls in the experiments. Also experiments in an ice channel with a varying width and with a varying depth were performed to study landing of aircraft on ice in frozen bays and straits $[17,25]$. It is mentioned also that the ice thickness is a very important parameter of experiments in ice channels. For ice thickness greater than $4 \mathrm{~mm}$, the presence of the vertical walls of the tank becomes important in the experiments described in [17], page 210.

A finite-element method was used in [23] to investigate the response of an ice sheet to moving loads. The hydrodynamic pressure acting on the lower surface of the ice was described by vertical modes of the channel, but the modes were not specified or explained. The linear problem of ice response was solved for each vertical mode. Calculations were performed for the ice tank of $10 \mathrm{~m}$ long and $4 \mathrm{~m}$ wide. The numerical results were compared with the results of experiments in terms of the ice deflection. The effect of the vertical walls of the channel on deflections was found to be significant. It was shown in [24] that the stresses and deflections of ice cover, which are caused by a load moving along the cover near vertical wall, strongly depend on the distance of the load trajectory from the wall.

The hydroelastic waves in a frozen channel were investigated by Daly [26] and by Steffler and Hicks [27] in the one-dimensional approximation. Three equations of mass and momentum conservation, and the ice cover response were used in linearized form. The ice cover was modelled by elastic beam. The type of connection of the ice cover to the channel walls was not included in this one-dimensional model.

To investigate the ice response in a channel for different speeds of the load, the critical speeds of the hydroelastic waves in the channel should be determined. For the one-dimensional model of ice cover in a channel $[26,27]$ and the ice plate of infinite extent [1], there is only one dispersion relation between frequency 
and the length of hydroelastic wave and, correspondingly, only one critical speed. For a channel covered with ice, there are infinitely many dispersion relations and corresponding critical speeds [28]. The problem of periodic progressive hydroelastic waves propagating along the channel was studied in [28, 29]. The ice cover clamped to the walls of the channel was studied in [28] and the free-free ice cover was considered in [29]. The wave frequencies of progressive hydroelastic waves and the wave profiles were determined for given wave length along the channel. The linear problem of hydroelasticity was reduced to the problem of wave profile across the channel. The problem was solved by the normal mode method. The dispersion relations and critical speeds of the propagating waves were determined. The first critical speed for the ice cover clamped to the channel walls was found to be slightly higher than that for the identical plate of infinite extent. It was concluded that strains reach their maximum at the walls for long waves and at the centre line of the channel for short waves. It was shown that the hydroelastic waves in the ice cover clamped to the walls propagate faster than for the free ice cover and are of higher frequency for the same wave length. In the present paper it will be shown that the critical speeds of the hydroelastic waves in a frozen channel help to understand and predict the response of the ice cover to a moving load depending on its speed, as it has been done for ice plate of infinite extent (see [1]).

It was observed $[30,31]$ that flexural waves, which are caused by a vehicle moving across a thin elastic plate, occur if the speed of the vehicle exceeds the minimum $c_{\min }$ of the phase speed of elastic-gravity free waves in the plate. The plate response is approximately quasi-static for lower speeds of the vehicle. It was concluded in [31] that "The amplified response at the critical speed $V=c_{\min }$ corresponds to an accumulation of energy underneath the source, since $c_{\text {min }}$ coinsides with the group speed." Some observed features of hydroelastic waves cannot be explained within the linear elastic theory: damping of waves with distance from the source [31] and the lag of the position of maximum depression immediately behind the source [32]. These effects are attributed to the viscoelastic damping of ice response [31, 32].

Viscoelastic properties of the sea ice were investigated by Tabata [33]. He performed experiments with rectangular ice beams depressed under static loads, and obtained stress-time curves. By analysing these curves Tabata concluded that "viscoelastic properties of sea ice are analogous to a rheological model of Maxwell unit and a Voigt (Kelvin) unit connected in series. Therefore, viscoelastic properties of sea ice can be expressed by four characteristic constants." A two-parameter viscoelastic model was used by Hosking et al. [31]. The Maxwell and Kelvin-Voigt models of viscoelastic ice were studied in [25]. The theoretical predictions of the ice response caused by a moving load within these two models and their combinations were compared with available experimental results. To improve the theoretical predictions, it was suggested to consider parameters in both models as functions of the speed of the load.

A Kelvin-Voigt model [34] of viscoelastic ice is used in this study. This is one of the simplest model of viscoelastic material. The constitutive equation of this model is $\sigma=E(\epsilon+\tau \partial \epsilon / \partial t)$, where $E$ is the Young's modulus, $\sigma$ is the stress, $\epsilon$ is the strain and $\tau$ is the so-called retardation time. The Kelvin-Voigt model of ice was used by Zhestkaya and Kozin[22, 23] and by Brocklehurst [35] (chapter 5) in the problems of load moving on ice sheet. The dissipation effects, which are characterized by the retardation time in the Kelvin-Voigt viscoelastic model of ice plate, were very strong in the numerical analysis by Zhestkaya and 
Kozin[23]. The retardation time was varied from 3 to 10 seconds in [23] to fit the experimental deflections. The experimental and numerical results of [23] showed that the magnitude of the stresses is dependent on the value of the retardation time but the critical speed of the ice plate is less sensitive to this value.

The stress-strain relation of the Kelvin-Voigt model is rather simplified (see [1] for more advanced models of viscoelastic ice). However, the present study is focused on the effect of the channel walls on the stresses in the ice cover of the channel. The simple Kelvin-Voigt model can be readily replaced with a more accurate one within the present approach.

The present paper is concerned with viscoelastic response of the ice cover in a channel caused by an external load moving with constant speed along the centreline of the channel. The ice cover is uniform through its thickness. The problem is considered in the coordinate system moving together with the load. The response of the ice cover is stationary in this system within a viscoelastic plate model. The problem is solved using the Fourier transform along the channel. The transformed function of the ice deflections is complex-valued due to the viscoelastic effect. The profile of the ice deflections across the channel is determined by the normal mode method for each value of the parameter of the Fourier transform. This approach leads to the linear algebraic system with respect to complex principal coordinates of the normal modes. The right-hand side of the system depends on the magnitude and distribution of the moving load. The matrix of this system was studied in [28] for zero retardation time. The eigenvalues of the matrix, which provide dispersion relations of the hydroelastic waves in the channel, and the corresponding eigen vectors, which provide the profiles of the hydroelastic waves across the channel, were determined and investigated. The system is truncated and solved numerically. The deflection of the ice cover and the bending stresses in it are obtained numerically by the inverse Fourier transform.

The influence of the physical parameters of the problem, such as the speed of moving load, the width of the channel, the damping coefficient and others on the hydroelastic response of the ice plate is studied. We assume that the ice plate breaks there and then, where and when the maximum strain in the plate approaches an yield value. The yield strain value, the retardation time and other characteristics of the ice are assumed to be determined from experiments for real ice conditions. It is shown that the highest strains in the ice cover occur for the speed of the moving load being close to the lowest critical speed of the hydroelastic waves in the channel. If the load speed is close to other critical speeds in the channel, the stresses in the ice cover are also amplified but their maxima are smaller than the maximum stress for the lowest critical speed within the viscoelastic model of ice.

It is shown that the dynamic component of the liquid pressure is very important for accurate prediction of ice-fluid interaction. Two simplified models are considered: dry-plate model, in which the presence of the liquid beneath the ice cover is neglected, and the hydrostatic model which does not account for the dynamic component of the liquid pressure. Both simplified models give wrong results for the hydroelastic waves in the frozen channel. The hydrostatic model can be used only for very low speeds of the moving load.

Obtained results can be used for developing technologies of both icebreaking between vertical walls and safe transportation on ice along frozen waterways.

The problem formulation and general assumptions are given in Section 2. The method of solution by 

reported and discussed in Section 4. Two simplified models are introduced and verified against the complete hydroelastic model in Section 5. The conclusion are drown in Section 6.

\section{Formulation of the problem}

The deflection of an ice sheet caused by the motion of an external load in the positive $x$-direction along a channel is considered. The channel is of rectangular cross section with depth $H$ and width $2 L$. The channel is of infinite extent in the $x$-direction (see Figure 1). The channel is occupied with the liquid of density $\rho_{l}$. The liquid is inviscid and incompressible. The liquid is covered with the ice sheet of constant thickness $h_{i}$ and rigidity $D=E h_{i}^{3} /\left[12\left(1-\nu^{2}\right)\right]$, where $E$ is Young's modulus of ice and $\nu$ is Poisson's ratio. The ice sheet is modelled by thin visco-elastic Kelvin-Voigt plate. The ice sheet is clamped to the walls of the channel at $y= \pm L$. The external load is modelled by a localised smooth pressure distribution over the upper surface of the ice sheet. The resulting deflection of the ice sheet, $z=w(x, y, t)$, is to be determined for the given load and given speed of its motion $U$ along the channel. The problem of deflection of the ice sheet in the channel is formulated within the linear theory of hydroelasticity [1]. The deflection of the ice sheet $w(x, y, t)$ is described by the equation of thin viscoelastic plate [34]

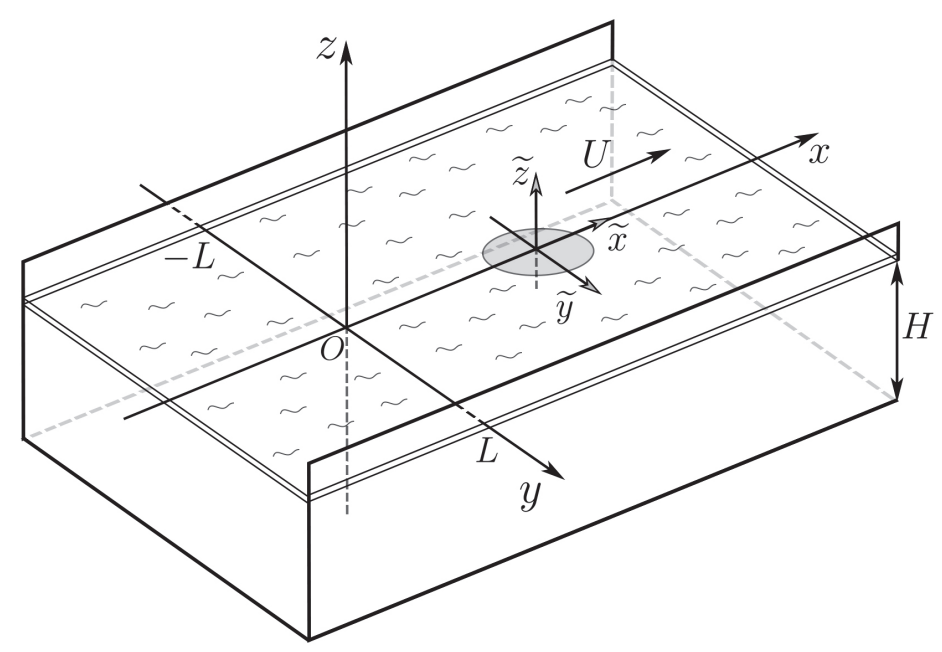

Figure 1: Sketch of the ice sheet in a channel with a load moving along the ice in the positive $x$-direction.

$$
\begin{gathered}
M w_{t t}+D\left(1+\tau \frac{\partial}{\partial t}\right) \nabla^{4} w=-P(x, y, t)+p(x, y, 0, t) \\
(-\infty<x<\infty,-L<y<L, z=0)
\end{gathered}
$$

where $\tau=\eta / E$ is the retardation time, $\eta$ is the viscosity of the ice, $\nabla^{4}=\partial^{4} / \partial x^{4}+2 \partial^{4} /\left(\partial x^{2} \partial y^{2}\right)+\partial^{4} / \partial x^{4}$, $M=\rho_{i} h_{i}$ is the mass of the ice plate per unit area, $\rho_{i}$ is the ice density, $p(x, y, 0, t)$ is the hydrodynamic pressure acting on the lower surface of the ice plate, $P(x, y, t)$ is the external pressure and $t$ is the time. The 
external pressure $P(x, y, t)$ moves along the central line of the channel and is described by

$$
\begin{gathered}
P(x, y, t)=P_{0} P_{1}\left(\frac{x-U t}{L}\right) P_{2}\left(\frac{y}{L}\right) \quad(-\infty<x<\infty,-L<y<L), \\
P_{1}(\widetilde{x})=(\cos (\pi c \widetilde{x})+1) / 2\left(c_{1}|\widetilde{x}|<1\right), \quad P_{1}(\widetilde{x})=0 \quad\left(c_{1}|\widetilde{x}| \geq 1\right), \quad \widetilde{x}=(x-U t) / L, \\
P_{2}(\widetilde{y})=(\cos (\pi k \tilde{y})+1) / 2\left(c_{2}|\widetilde{y}|<1\right), \quad P_{2}(\widetilde{y})=0 \quad\left(c_{2}|\widetilde{y}| \geq 1\right), \quad \widetilde{y}=y / L,
\end{gathered}
$$

where $c_{1}$ and $c_{2}$ are non-dimensional parameters of the external load characterizing the size of the pressure area. We restrict ourselves here to symmetric loads, however, the analysis can be readily extended to loads moving closer to one of the walls and to asymmetric loads.

The hydrodynamic pressure on the ice-liquid interface is given by the linearised Bernoulli equation,

$$
p(x, y, 0, t)=-\rho_{l} \varphi_{t}-\rho_{l} g w \quad(-\infty<x<\infty,-L<y<L),
$$

where $g$ is the gravitational acceleration and $\varphi(x, y, z, t)$ is the velocity potential of the flow beneath the ice cover. The velocity potential $\varphi(x, y, z, t)$ satisfies Laplace's equation in the flow region and the boundary conditions

$$
\varphi_{z}=w_{t} \quad(z=0), \quad \varphi_{y}=0 \quad(y= \pm L), \quad \varphi_{z}=0 \quad(z=-H)
$$

The ice sheet is frozen to the walls, which is modelled by the clamped conditions

$$
w=0, \quad w_{y}=0 \quad(-\infty<x<\infty, y= \pm L)
$$

The term with $\tau \partial / \partial t$ in the equation of viscoelastic plate (1) describes the damping of ice plate oscillations, so hydroelastic waves decay far away from the moving load, where $|\widetilde{x}| \rightarrow \infty$.

The formulated problem is considered in non-dimensional variables denoted by tilde. The half-width of the channel $L$ is taken as the length scale, the ratio $L / U$ as the time scale, and the pressure magnitude $P_{0}$ as the pressure scale. The non-dimensional depth of the channel $H / L$ is denoted by $h$. The moving coordinate system $(\widetilde{x}, \widetilde{y}, \widetilde{z})$ with the origin at the centre of the load is introduce by

$$
\widetilde{y}=\frac{y}{L}, \quad \widetilde{x}=\frac{x-U t}{L}, \quad \widetilde{z}=\frac{z}{L}, \quad \widetilde{t}=\frac{U}{L} t, \quad \widetilde{P}=P_{1}(\widetilde{x}) P_{2}(\widetilde{y})
$$

We are concerned with a steady-state solution in the moving coordinate system,

$$
\begin{aligned}
& w(x, y, t)=w(\widetilde{x} L+U t, L \widetilde{y}, t)=w_{s c} \widetilde{w}(\widetilde{x}, \widetilde{y}), \\
& \varphi(x, y, t)=\varphi(\widetilde{x} L+U t, L \widetilde{y}, t)=\varphi_{s c} \widetilde{\varphi}(\widetilde{x}, \widetilde{y}, \widetilde{z}),
\end{aligned}
$$

where $w_{s c}$ and $\varphi_{s c}$ are the scales of the ice deflection and the velocity potential correspondingly. The scales are chosen as $w_{s c}=P_{0} /\left(\rho_{l} g\right)$ and $\varphi_{s c}=\left(U P_{0}\right) /\left(\rho_{l} g\right)$.

In the non-dimensional variables the problem reads (tildes are omitted below)

$$
\begin{gathered}
\alpha h \operatorname{Fr}^{2} w_{x x}+\beta\left(1-\varepsilon \frac{\partial}{\partial x}\right) \nabla^{4} w+w=h \operatorname{Fr}^{2} \varphi_{x}-P_{1}(x) P_{2}(y) \\
(-\infty<x<\infty, \quad-1<y<1, \quad z=0),
\end{gathered}
$$




$$
\begin{gathered}
\nabla^{2} \varphi=0 \quad(-\infty<x<\infty, \quad-1<y<1, \quad-h<z<0), \\
\varphi_{z}=-w_{x} \quad(z=0), \quad \varphi_{y}=0 \quad(y= \pm 1), \quad \varphi_{z}=0 \quad(z=-h), \\
w=0, \quad w_{y}=0 \quad(y= \pm 1), \\
w, \varphi \rightarrow 0 \quad(|x| \rightarrow \infty) .
\end{gathered}
$$

Here $\beta=D /\left(\rho_{l} g L^{4}\right), \varepsilon=(\tau U) / L, \alpha=\left(\rho_{i} h_{i}\right) /\left(\rho_{l} L\right)$ and $\mathrm{Fr}=U / \sqrt{g H}$ is the Froude number.

The solution of the problem (6)-(10) depends on seven non-dimensional parameters $h, \alpha, \beta, \varepsilon, \operatorname{Fr}, c_{1}$ and $c_{2}$ which describe the aspect ratio of channel, characteristics of ice and of the applied load. We shall determine the deflection $w$ and strain distribution in the ice sheet for some given values of these parameters.

In the linear theory of hydroelasticity, the strains vary linearly through the ice thickness being zero at the middle of the plate thickness. At any location, the maximum strain is achieved at the surface of the ice. We are concerned only with positive strains which correspond to elongation of the ice surface and tensile stresses in the ice. The scale of the strains is taken as $h_{i} P_{0} /\left(2 \rho_{l} g L^{2}\right)$. The strain tensor is given by

$$
E(x, y)=-\zeta\left(\begin{array}{ll}
w_{x x} & w_{x y} \\
w_{x y} & w_{y y}
\end{array}\right),
$$

where $\zeta$ is the non-dimensional coordinate across the ice thickness, $-1 \leq \zeta \leq 1$. The tensor (11) describes the strain field in the ice sheet. To find the maximum strain in the ice sheet we need to find the eigenvalues of the strain tensor at each location. The strains are proportional to the magnitude $P_{0}$ of the external load within the linear theory. The linear theory of hydroelasticity can be used when $w_{x}^{2}+w_{y}^{2}$ is small and the strains in the ice sheet are below the yield strain $\epsilon_{c r}$ of the ice.

The yield strength of a material is defined as the strain $\epsilon=\epsilon_{c r}$ at which a material begins to deform plastically [36]. We require that the strains in the ice sheet are below the yield strain of ice, to prevent our visco-elastic model from becoming unrealistic; any strains greater than the yield strain $\epsilon_{c r}$ are assumed to lead to ice fracture. Squire [37] studied break-up of sea ice within a linear viscoelastic model. The theoretical predictions were found to be in agreement with the experimental results by Goodman et al. [38], who measured the strains in sea ice with the wire strainmeter. The observed fracture strain was reported as $3 \cdot 10^{-5}$, and the theory predicts ice fracture if the strain reaches $4.3 \cdot 10^{-5}$. Squire and Martin [39] determined the fracture strain for Bering Sea ice to be $4.4-8.5 \cdot 10^{-5}$. In this study we use the estimate $\epsilon_{c r}=8 \cdot 10^{-5}$ (see [24] and discussion of this value there).

The formulation of the problem (6)-(10) can be related to the problem of progressive hydroelastic waves in a frozen channel, which was studied in [28]. Consider a wave with a frequency $\omega$ and a wave number $k$. In the coordinate system moving at the phase speed, $U=\omega / k$, the hydroelastic wave is stationary, $w(x, y)$, and periodic along the channel, $w(x, y)=F(y, k) \cos (k L x)$. Substituting this form of the deflection in (6)-(9), setting $\varepsilon=0$, and omitting both the far-field condition (10) and the external load, we arrive at the eigen value problem with respect to the wave profile across the channel, $F(y, k)$, and the dispersion relations between the wave frequency $\omega$ and the wave number $k$. There are infinite number of such dispersion relations, $\omega=\omega_{n}(k)$, where $n \geq 1$ and $\omega_{n+1}(k)>\omega_{n}(k)$ for $k>0$, and infinite number of the corresponding hydroelastic waves 
with the profiles $F_{n}(y, k)[28]$. In the present problem with stationary deflection in the moving coordinate system, only progressive hydroelastic waves with phase speed equal to $U$ contribute to the ice deflection. For each value of the load speed $U$ only a finite number of hydroelastic waves and only the waves with certain wave numbers contribute to the stationary ice deflection (see figure $6 \mathrm{~b}$ in [28]). This relation between the present problem and progressive hydroelastic waves will be used in section 4 for interpretation of the obtained numerical results for the deflection of the ice cover near the moving load. Below the hydroelastic wave with the dispersion relation $\omega=\omega_{n}(k)$ is referred to as the $n$-th wave propagating along the channel.

\section{Solution of the problem}

The coupled problem (6)-(10) is solved with the help of the Fourier transform in the $x$ direction. The plate equation (6) provides

$$
\left(1-\alpha h \operatorname{Fr}^{2} \xi^{2}\right) w^{F}+\beta(1-i \xi \varepsilon)\left(w_{y y y y}^{F}-2 \xi^{2} w_{y y}^{F}+\xi^{4} w^{F}\right)=i \xi h \operatorname{Fr}^{2} \varphi^{F}-P^{F}(\xi, y)
$$

where

$$
w^{F}(\xi, y)=\frac{1}{\sqrt{2 \pi}} \int_{-\infty}^{\infty} w(x, y) e^{-i \xi x} d x, \quad P^{F}(\xi, y)=P_{2}(y) P_{1}^{F}(\xi) .
$$

It is convenient to introduce the eigen modes $\psi_{j}(y)$ of one-dimensional clamped beam and seek $w^{F}(\xi, y)$ in the form of the series

$$
w^{F}(\xi, y)=\sum_{j=1}^{\infty} a_{j}(\xi) \psi_{j}(y)
$$

with the coefficients $a_{j}(\xi)$ to be determined. The decomposition (13) was used in [28] to determine the dispersion relations of hydroelastic waves propagating along the frozen channel. The modes $\psi_{j}(y)$ are nonzero solutions of the eigen-value problem

$$
\psi_{j}^{I V}=\lambda_{j}^{4} \psi_{j} \quad(-1<y<1), \quad \psi_{j}=\psi_{j}^{\prime}=0 \quad(y= \pm 1) .
$$

In the present problem of a symmetric load moving along the central line of the channel, the solution is even in $y$, so we need only the even modes $\psi_{j}(y)$ :

$$
\psi_{j}(y)=A_{j}\left(\cos \lambda_{j} y-B_{j} \cosh \lambda_{j} y\right), \quad B_{j}=\frac{\cos \lambda_{j}}{\cosh \lambda_{j}}, \quad A_{j}^{2}\left(1+B_{j}^{2}\right)=1,
$$

where $\lambda_{j}$ are the solutions of the equation $\tan \lambda_{j}=-\tanh \lambda_{j}$. It can be shown that $\lambda_{j}=\pi j-\pi / 4+\Delta_{j}$, where $\Delta_{j} \rightarrow 0$ as $j \rightarrow \infty$. The modes (15) are orthonormal.

Substituting series (13) in the equation (12), multiplying both sides of the equation by $\psi_{m}(y), m \geq 1$, integrating the result in $y$ from -1 to 1 , and using (14), we arrive at the infinite system of algebraic equations

$$
\begin{gathered}
\left(1-\alpha h \operatorname{Fr}^{2} \xi^{2}\right) a_{m}+\beta(1-i \xi \varepsilon)\left(\lambda_{m}^{4} a_{m}-2 \xi^{2} \sum_{j=1}^{\infty} C_{m j} a_{j}+\xi^{4} a_{m}\right)= \\
=\xi^{2} h \operatorname{Fr}^{2} \sum_{j=1}^{\infty} M_{m j} a_{j}-P_{m}(\xi)
\end{gathered}
$$




$$
\begin{gathered}
C_{m j}=-\int_{-1}^{1} \psi_{m}^{\prime}(y) \psi_{j}^{\prime}(y) d y, \quad M_{m j}(\xi)=\int_{-1}^{1} \phi_{j}(y, 0, \xi) \psi_{m}(y) d y, \\
\varphi^{F}(\xi, y, z)=\sum_{j=1}^{\infty} a_{j}(\xi) \phi_{j}(y, z, \xi), \quad P_{m}(\xi)=P_{1}^{F}(\xi) P_{m}^{*}, \quad P_{m}^{*}=\int_{-1}^{1} P_{2}(y) \psi_{m}(y) d y .
\end{gathered}
$$

The functions $\phi_{j}(y, z, \xi)$ are the solutions of the following boundary-value problems

$$
\begin{gathered}
\phi_{j, y y}+\phi_{j, z z}=\xi^{2} \phi_{j} \quad(-1<y<1,-h<z<0), \\
\phi_{j, y}=0 \quad(y= \pm 1), \quad \phi_{j, z}=0 \quad(z=-h), \quad \phi_{j, z}=\psi_{j}(y) \quad(z=0) .
\end{gathered}
$$

The variable $\xi$ of the Fourier transform plays the role of a parameter here. The integrals $C_{m j}$ and $M_{m j}(\xi)$ were calculated in [28]. Coefficients $P_{m}^{*}$ are evaluated analytically for the function $P_{2}(y)$ given by (2).

The system (16) can be written in the matrix form

$$
\begin{gathered}
\mathbf{A} \vec{a}=-\vec{P}, \\
\mathbf{A}=\left(1-\alpha h \operatorname{Fr}^{2} \xi^{2}\right) \mathbf{I}+\beta(1-i \xi \varepsilon) \mathbf{Q}-h \operatorname{Fr}^{2} \xi^{2} \mathbf{M},
\end{gathered}
$$

220

where $\vec{a}=\left(a_{1}, a_{2}, \ldots\right)^{T}, \mathbf{M}=\left\{M_{m j}\right\}_{m, j=1}^{\infty}, \vec{P}=\left(P_{1}, P_{2}, \ldots\right)^{T}, \mathbf{Q}=\mathbf{D}-2 \xi^{2} \mathbf{C}$ and $\mathbf{D}=\operatorname{diag}\left\{\lambda_{1}^{4}+\xi^{4}, \lambda_{2}^{4}+\right.$ $\left.\xi^{4}, \ldots\right\}, \mathbf{C}=\left\{C_{m j}\right\}_{m, j=1}^{\infty}$. The eigen values of the matrix $\mathbf{A}$ with $\varepsilon=0$ were studied in [28]. These eigen values provide the dispersion relations of hydroelastic waves in the channel.

To solve equation (17) we distinguish the real and imaginary parts of the vector $\vec{a}, \vec{a}=\vec{a}^{R}+i \vec{a}^{I}$. Note that all other vectors and elements of the matrices in (17) are real, which provides the system of nonhomogeneous equations with respect to $\vec{a}^{R}$ and $\vec{a}^{I}$ with symmetric matrices. In the present problem, $P_{1}^{F}(\xi)$ is an even function of $\xi$. It can be shown that $a_{j}^{R}(\xi)$ are even and $a_{j}^{I}(\xi)$ are odd functions of $\xi$.

The deflection $w(x, y)$ is obtained by the inverse Fourier transform

$$
w(x, y)=\sqrt{\frac{2}{\pi}} \sum_{j=1}^{\infty} \psi_{j}(y) \int_{0}^{\infty}\left(a_{j}^{R}(\xi) \cos (\xi x)-a_{j}^{I}(\xi) \sin (\xi x)\right) d \xi,
$$

where the functions $a_{j}^{R}(\xi)$ and $a_{j}^{I}(\xi)$ are calculated as solutions of (17) at $\xi=\xi_{n}, 1 \leq n \leq N_{\xi}, \xi_{1}=0$, with a step $\Delta \xi$.

The infinite series in (18) is truncated to $N_{m o d}$ terms and the integrals are approximated by

$$
\begin{gathered}
w(x, y) \approx \sqrt{\frac{2}{\pi}} \sum_{j=1}^{N_{\text {mod }}} \psi_{j}(y) W_{j}(x), \\
W_{j}(x)=\sum_{n=1}^{N_{\xi}} \int_{\xi_{n}}^{\xi_{n+1}}\left(a_{j}^{R}(\xi) \cos (\xi x)-a_{j}^{I}(\xi) \sin (\xi x)\right) d \xi .
\end{gathered}
$$

The integrals in (20) are evaluated by using the linear interpolations of $a_{j}^{R}(\xi)$ and $a_{j}^{I}(\xi)$ in each interval $\left[\xi_{n}, \xi_{n+1}\right]$.

In order to investigate the dependences of the deflections of the ice cover and stresses in it on the width of the channel, the obtained results are compared with the corresponding results for the ice sheet of infinite 
extent. The corresponding solution $w_{\infty}(x, y)$ for the infinite ice plate and the same external load (2) is obtained by applying the Fourier transform in $y$ to equation (12). Then

$$
\begin{aligned}
w_{\infty}(x, y)=\frac{2}{\pi} \int_{0}^{\infty} & \left(\int_{0}^{\infty} Q^{R}(\xi, \eta) \cos (\eta y) d \eta\right) \cos (\xi x) d \xi- \\
& -\frac{2}{\pi} \int_{0}^{\infty}\left(\int_{0}^{\infty} Q^{I}(\xi, \eta) \cos (\eta y) d \eta\right) \sin (\xi x) d \xi
\end{aligned}
$$

where

$$
\begin{gathered}
Q^{R}(\xi, \eta)=\frac{-P^{X Y} q^{R}}{\left(q^{R}\right)^{2}+\left(q^{I}\right)^{2}}, \quad Q^{I}(\xi, \eta)=\frac{P^{X Y} q^{I}}{\left(q^{R}\right)^{2}+\left(q^{I}\right)^{2}}, \quad P^{X Y}(\xi, \eta)=P_{1}^{F}(\xi) P_{2}^{F}(\eta), \\
q^{R}=1-\alpha h \mathrm{Fr}^{2} \xi^{2}-\frac{\xi^{2} h \mathrm{Fr}^{2} \operatorname{coth}(\lambda h)}{\lambda}+\beta \lambda^{4}, \quad q^{I}=-\xi \varepsilon \lambda^{4}, \quad \lambda=\sqrt{\xi^{2}+\eta^{2}} .
\end{gathered}
$$

The integrals in (21) are evaluated in the same way as the integrals in (18).

\section{Numerical results}

The hydroelastic behaviour of the ice cover in a channel, which is caused by a moving load, is investigated

numerically for a freshwater ice with density $\rho_{i}=917 \mathrm{~kg} / \mathrm{m}^{3}$, Young's modulus $E=4.2 \cdot 10^{9} \mathrm{~N} / \mathrm{m}^{2}$, Poisson's ratio $\nu=0.3$ and the retardation time $\tau=0.1 \mathrm{~s}$. The thickness of the ice plate is $10 \mathrm{~cm}$ and the water depth is $2 \mathrm{~m}$. The load (2) is applied over the area $2 \mathrm{~m} \times 2 \mathrm{~m}$ and moves along the central line of the channel at constant speed. The speed of the load, $U$, and the half-width of the channel, $L$, vary in the calculations. The ranges of the speeds $U$ and the widths $L$ are chosen according to the critical speeds of hydroelastic waves propagating in the frozen channel, which were computed in [28], and the characteristic length $\left(D / \rho_{l} g\right)^{1 / 4}$ of the ice cover, which is equal to $2.48 \mathrm{~m}$ in the case under consideration.

The calculations of the ice deflection, $w(x, y)$, and its second derivatives in the strain tensor (11) require numerical evaluation of the integrals in (18). The number of these integrals depends on the number of retained modes, $N_{\text {mod }}$. The step of integration, $\Delta \xi$, and the number of the steps, $N_{\xi}$, in (20) should be carefully assessed. The linear system (17) of $N_{\text {mod }}$ equations is solved $N_{\xi}$ times for each case. It was found that the external load (2) is accurately approximated by formulae similar to (19) and (20) with $N_{\bmod }=10$, $N_{\xi}=400$ and $\Delta \xi=0.25$ for $c_{1}=c_{2}=10$, that is, for the dimensions of the load area being ten times smaller than the width of the channel. In calculations of the ice deflection, the number of modes, $N_{\text {mod }}$, is varied from 5 to 15 , to confirm convergence of the numerical solutions.

The step of integration, $\Delta \xi$, and the number of steps, $N_{\xi}$, in (20) depend on the non-dimensional parameters of the problem, in particular, on the size of the load area and on the retardation time $\tau$. The values $\Delta \xi=0.25$ and $N_{\xi}=400$ provide accurate results for $c_{1}=c_{2}=10$ and $\tau=0.1 \mathrm{~s}$. These values were used in most of the presented calculations. The numerical analysis aims to investigate the ice deflection and strains in the ice sheet depending on the width of the channel and the speed of the load. The deflections and strains are presented below in the moving coordinate system with $x=0$ at the centre of the load. 

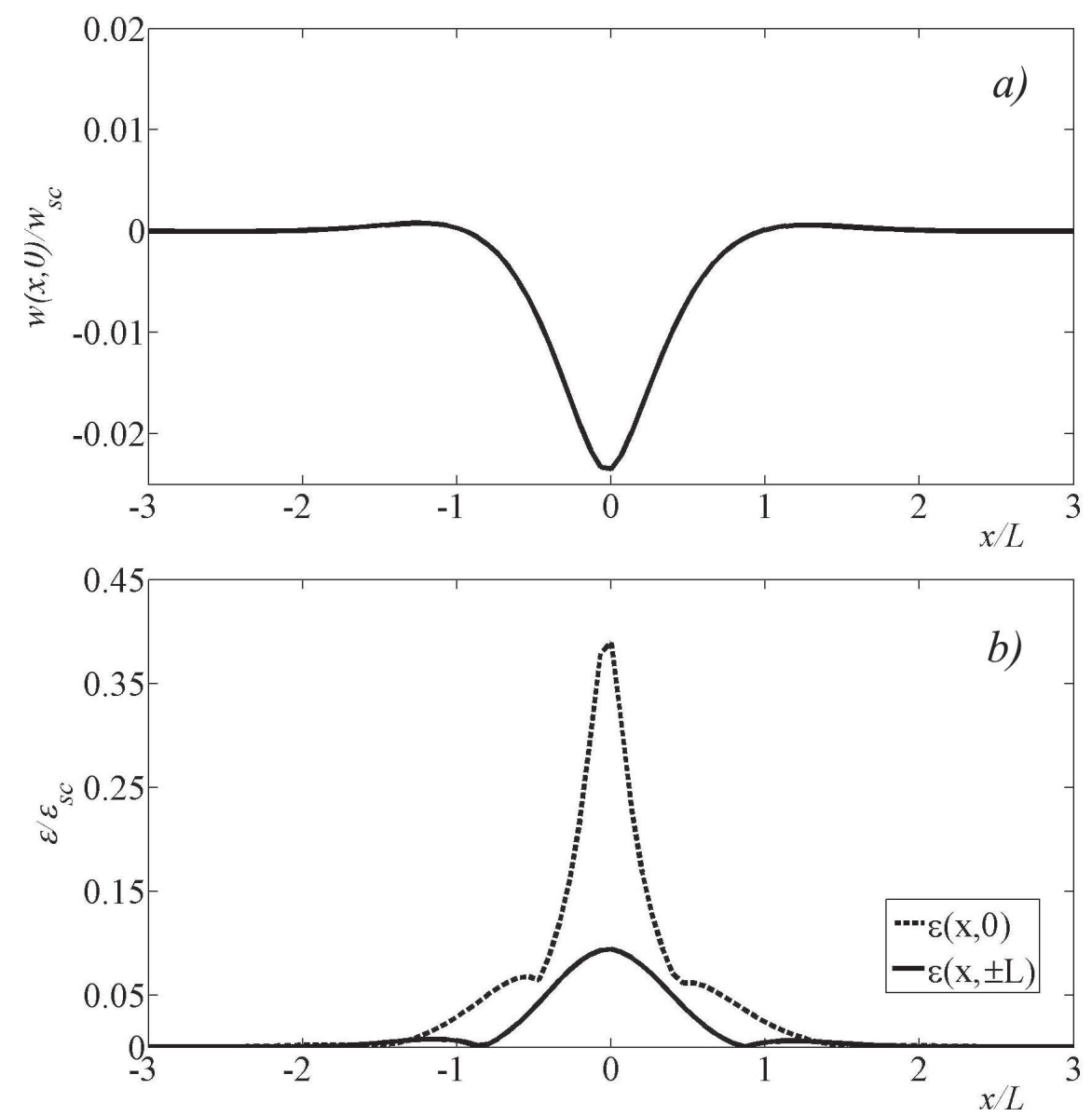

Figure 2: The deflection of the ice plate at the central line of the channel $(a)$ and strains $(b)$ for the load moving with the subcritical speed $U=3 \mathrm{~m} / \mathrm{s}$. Here $L=10 \mathrm{~m}, h_{i}=10 \mathrm{~cm}$ and $H=2 \mathrm{~m}$. The strains at the centre line are shown by the dotted line and the strains at the walls are shown by the solid line in $(b)$.

The nondimensional deflection of the ice sheet and the strain distributions along the central line of the channel, $y=0$, and along the walls, $y= \pm L$, are shown in Figures 2 and 3 as functions of the longitudinal coordinate $x / L$ along the channel of width $20 \mathrm{~m}$. The critical speed of the first hydroelastic wave $(n=1)$ for this width of the channel is $5.38 \mathrm{~m} / \mathrm{s}$ (see [28]). Subcritical case with the speed of the load $U=3 \mathrm{~m} / \mathrm{s}$ is shown in Figure 2 and the supercritical case with $U=7 \mathrm{~m} / \mathrm{s}$ is shown in Figure 3. The load moves from left to right. For the subcritical speed (Figure 2) the ice response is localised near the load, because the hydroelastic waves with phase speed below the critical speed do not exist (see [1] for the infinite ice plate and [28]) Fig 6 for the channel). The hydroelastic waves contribute to the ice response for supercritical speed (Figure 3). For the load speed $U=7 \mathrm{~m} / \mathrm{s}$, only the first hydroelastic wave $(n=1)$ with the wave number $k \approx 0.43 \mathrm{~m}^{-1}$ has phase speed equal to the load speed (see Fig. $6 \mathrm{~b}$ in [28]). This wave number corresponds to the wave length of $14.6 \mathrm{~m}$ which is equal to the distance between two subsequent peaks of ice deflection in Figure 3. Note that the module of the strains is shown in Figure 3b. Therefore, the load moving at $U=7 \mathrm{~m} / \mathrm{s}$ generates the first hydroelastic wave which is stationary in the moving coordinate 
system and the amplitude of which decay with the distance from the load due to the viscoelasticity of the ice. The deflections and strains in the plate are very small at the distance $20 \mathrm{~m}$ behind the load in both cases. The maximum strain is achieved on the central line in the place of the load for the subcritical speed but on the wall for the supercritical speed of the load. The strains are scaled with $\varepsilon_{s c}=h_{i} P_{0} /\left(2 \rho_{l} g L^{2}\right)$. For the yield strain $8 \times 10^{-5}$ (see [35]), we calculate that the ice is likely to fracture for $U=3 \mathrm{~m} / \mathrm{s}$ in the area of the load for the load magnitude $P_{0}$ greater that $4 \mathrm{kPa}$. Correspondingly for $U=7 \mathrm{~m} / \mathrm{s}$ (see Figure 3 ) the ice is likely to break from the wall but also for $P_{0}$ greater than $4 \mathrm{kPa}$.
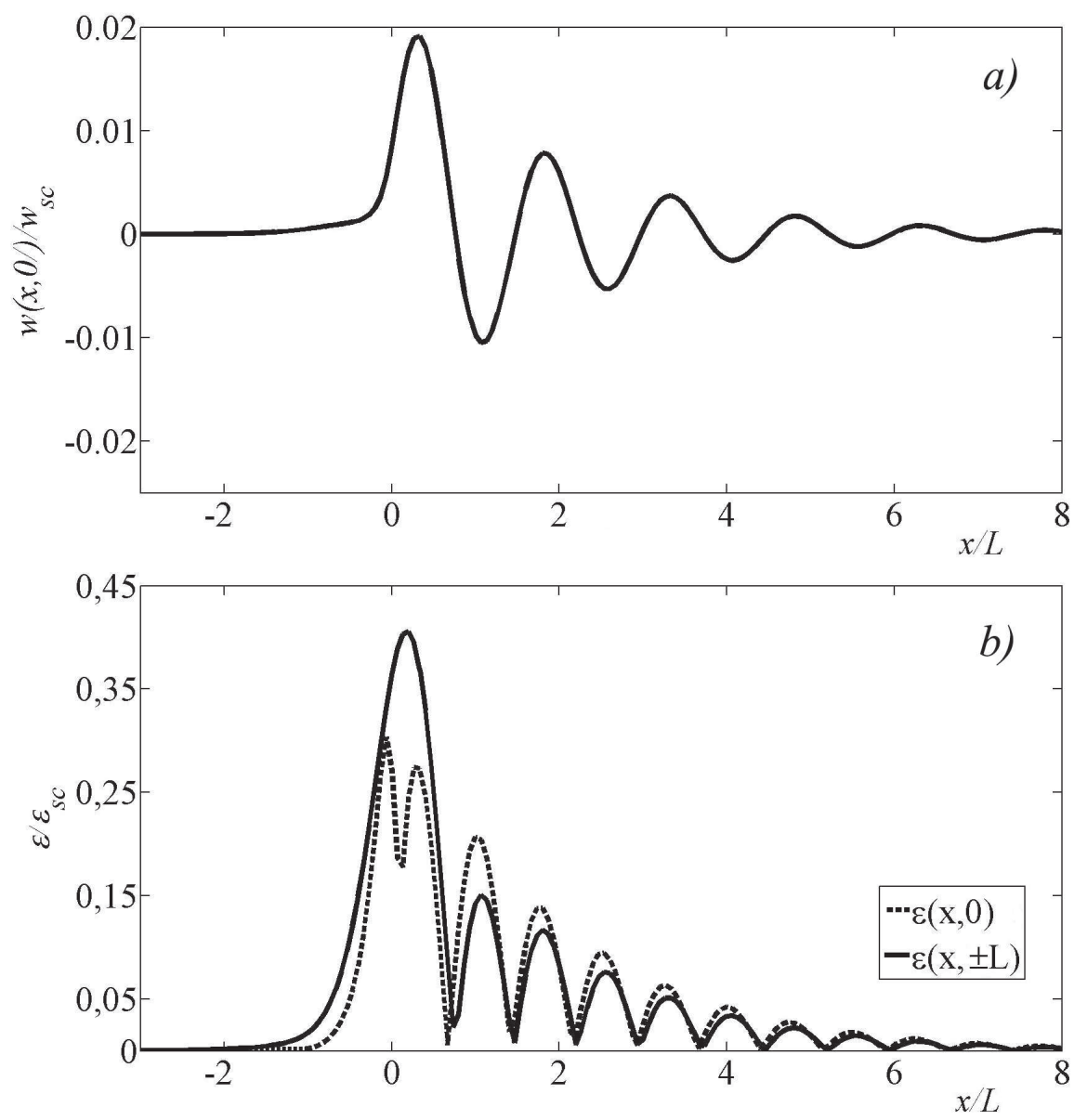

Figure 3: The deflection of the ice plate at the central line of the channel $(a)$ and strains $(b)$ for the load moving with the supercritical speed $U=7 \mathrm{~m} / \mathrm{s}$. Here $L=10 \mathrm{~m}, h_{i}=10 \mathrm{~cm}$ and $H=2 \mathrm{~m}$. The strains at the centre line are shown by the dotted line and the strains at the walls are shown by the solid line in $(b)$. 

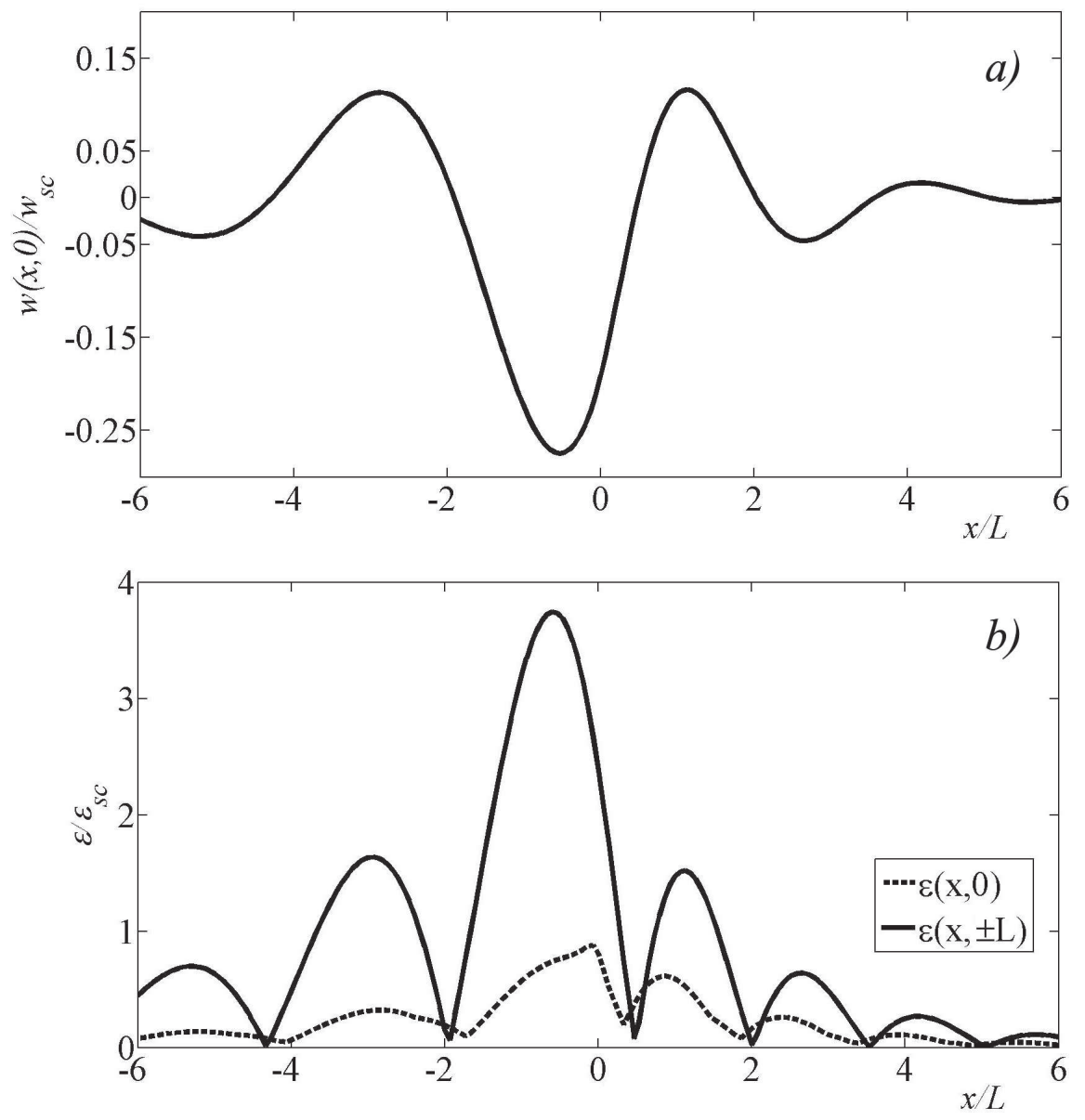

Figure 4: The deflection of the ice plate at the central line of the channel $(a)$ and strains $(b)$ for the load moving at the critical speed $U=5.38 \mathrm{~m} / \mathrm{s}$. Here $L=10 \mathrm{~m}, h_{i}=10 \mathrm{~cm}$ and $H=2 \mathrm{~m}$. The strains at the centre line are shown by the dotted line and the strains at the walls are shown by the solid line in $(b)$.

The nondimensional deflection of the ice at the central line and the strains at both the central line and along the walls for the critical speed of the first hydroelastic wave [28], $U=5.38 \mathrm{~m} / \mathrm{s}$, are shown in Figure 4 (compare the vertical scales in Figures 2, 3 and Figure 4). Both deflections and strains are about ten times larger for the critical speed of the load then for the subcritical, $U=3 \mathrm{~m} / \mathrm{s}$, and supercritical, $U=7 \mathrm{~m} / \mathrm{s}$ speeds. The maximum strain at the wall is about four times larger than at the central line (Figure $4 b)$. The ice plate oscillates both in front and behind the moving load. The strains at the wall exceed the yield strain for $P_{0}>206 \mathrm{~Pa}$. 

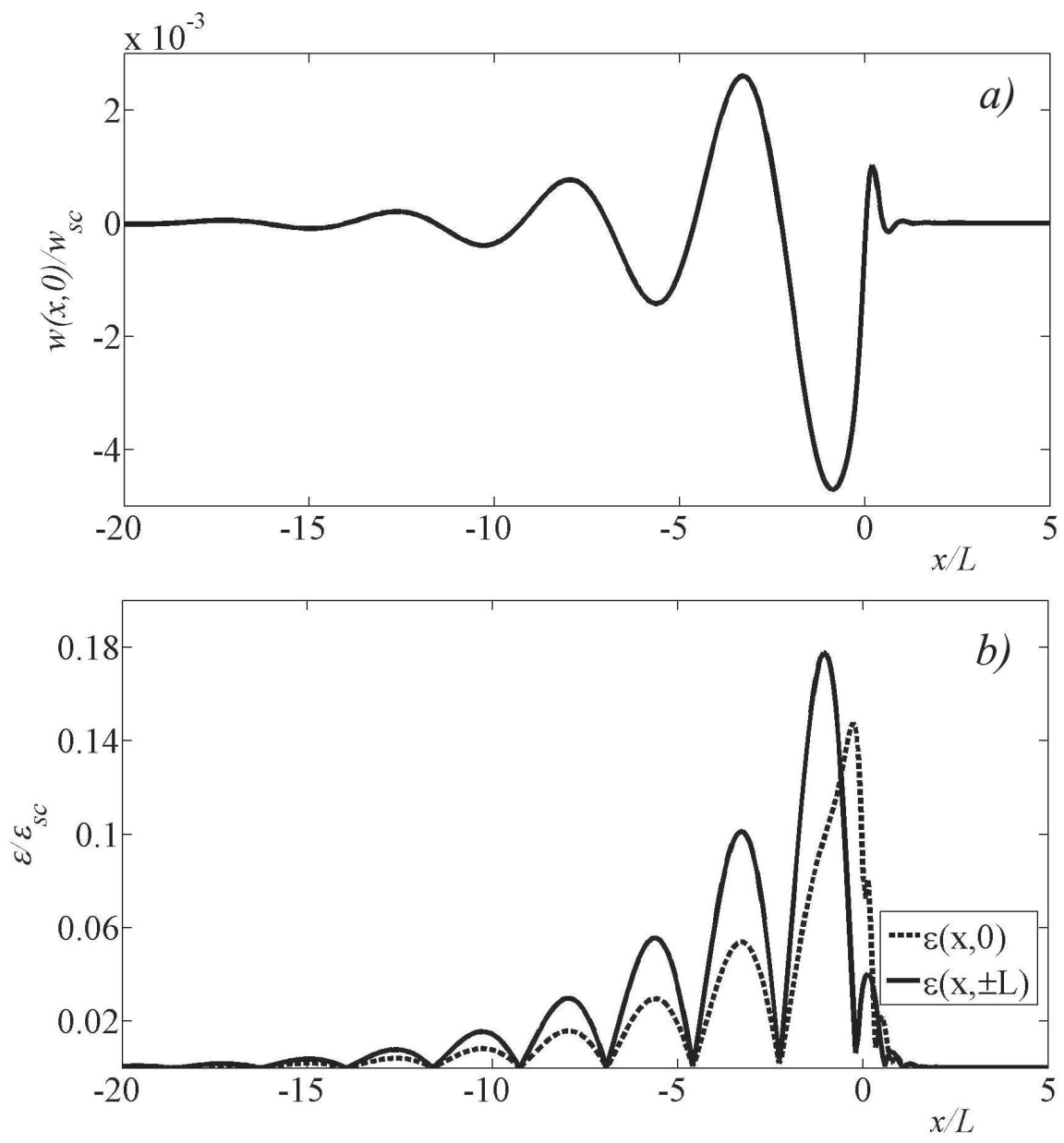

Figure 5: The deflection of the ice plate at the central line of the channel $(a)$ and strains $(b)$ for the load moving at the supercritical speed $U=25 \mathrm{~m} / \mathrm{s}$. Here $L=10 \mathrm{~m}, h_{i}=10 \mathrm{~cm}$ and $H=2 \mathrm{~m}$. The strains at the centre line are shown by the dotted line and the strains at the walls are shown by the solid line in $(b)$.

The deflection and the strains for the speed $U=25 \mathrm{~m} / \mathrm{s}$, are shown in Figure 5 . This speed is higher then the critical speed, $U \approx 15.3 \mathrm{~m} / \mathrm{s}[28]$, of the second even hydroelastic wave. There are no oscillations of the ice sheet in front of the moving load but they present behind the load. The strains achieve their maximum at the wall but this maximum is 20 times smaller than for the critical speed of the first hydroelastic wave (see Figure $4 \mathrm{~b}$ ). There are three hydroelastic waves with the phase speed $25 \mathrm{~m} / \mathrm{s}$ : the second hydroelastic wave with the wave numbers $k \approx 0.135 \mathrm{~m}^{-1}$ and $k \approx 1.02 \mathrm{~m}^{-1}$, and the first hydroelastic wave with the wave number $k \approx 1.2 \mathrm{~m}^{-1}$. The corresponding wave lengths are $45 \mathrm{~m}, 6.3 \mathrm{~m}$ and $6 \mathrm{~m}$. The ice plate oscillations behind the load correspond to the long wave of length $45 \mathrm{~m}$. The short waves can be observed in front of the load. 

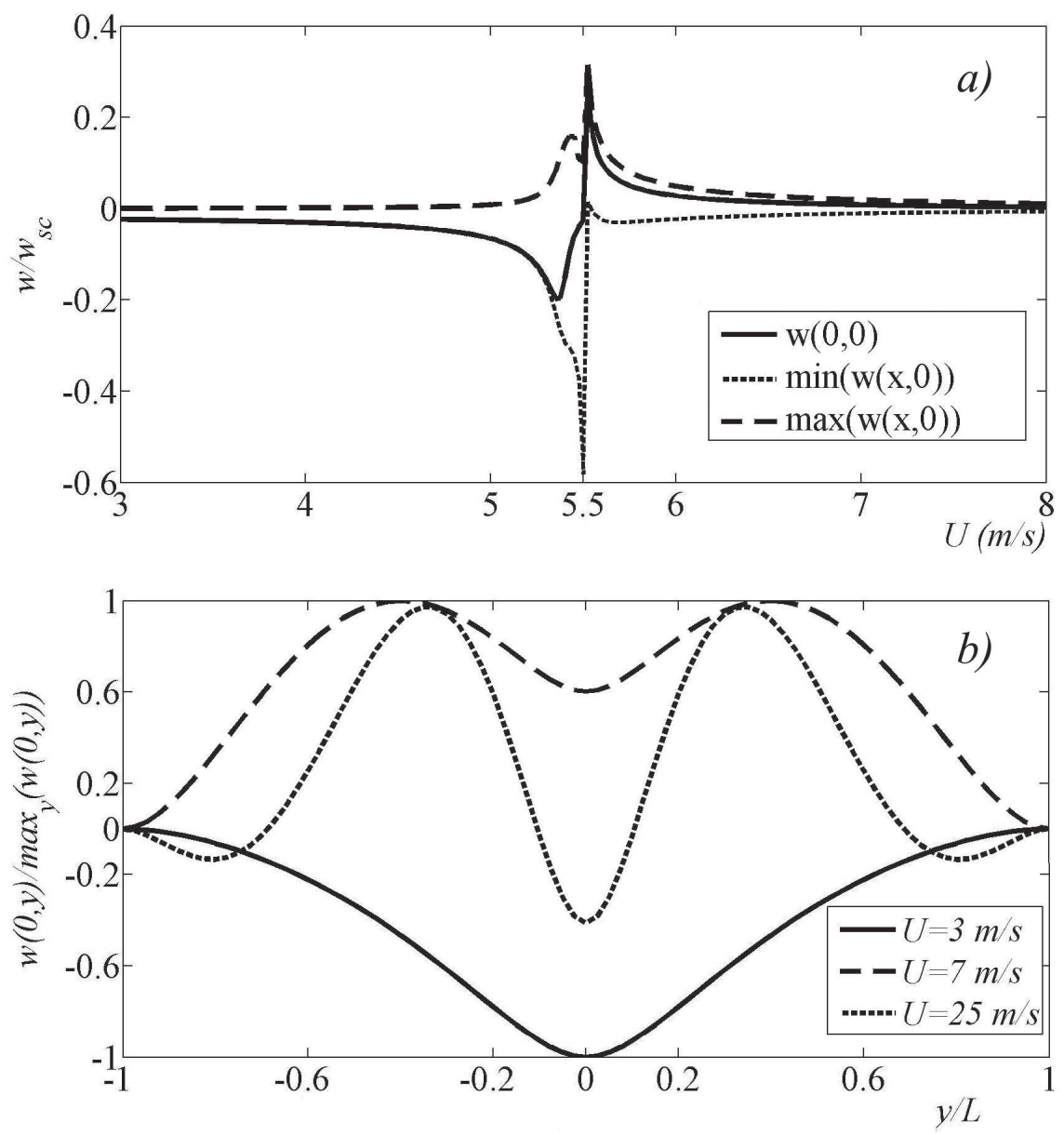

Figure 6: (a) The deflections at the centre of the load (solid line), the maximum (dashed line) and the minimum (dotted line) of the deflections along the central line of the channel are shown as functions of the load speed $U$; $(b)$ the normalized profiles of the ice plate across the channel at $x=0$ for $U=3,7$ and $25 \mathrm{~m} / \mathrm{s}$.

The nondimensional deflections at the central line of the channel are shown as functions of the load speed $U$ in Figure $6 a$. The deflection at the centre of the load, $x=y=0$, and both the minimum and maximum of the deflections along the central line $y=0$ are depicted. The deflections peak at $U \approx 5.5 \mathrm{~m} / \mathrm{s}$ which is slightly higher than the critical speed of the first hydroelastic wave, $5.38 \mathrm{~m} / \mathrm{s}$ from [28]. This could be due to the damping in the ice plate described by the parameter $\tau$, which is $0.1 \mathrm{~s}$ in Figures $2-6$. The critical speed of the second hydroelastic wave propagating along the channel and even in $y, U=15.3 \mathrm{~m} / \mathrm{s}$, was also detected but only in calculations with a very small retardation time, $\tau=0.001 \mathrm{~s}$.

The normalized profiles of the ice deflection across the channel at $x=0$ are shown in Figure $6 b$ for different speeds of the load. The main contribution to the profile corresponding to the subcritical speed, $U=3 \mathrm{~m} / \mathrm{s}$, comes from the profile of the first hydroelastic wave (see [28], figure 7). For the speed $U=7 \mathrm{~m} / \mathrm{s}$, which is higher than the critical speed of the first mode but below the critical speed of the second wave, the main contributions to the profile come from both the first and second hydroelastic wave profiles. For the speed $U=25 \mathrm{~m} / \mathrm{s}$, which is higher than the critical speed of the second wave, three first hydroelastic waves 
profiles $(n=1,2,3)$ contribute to the stationary profile of the ice deflection.
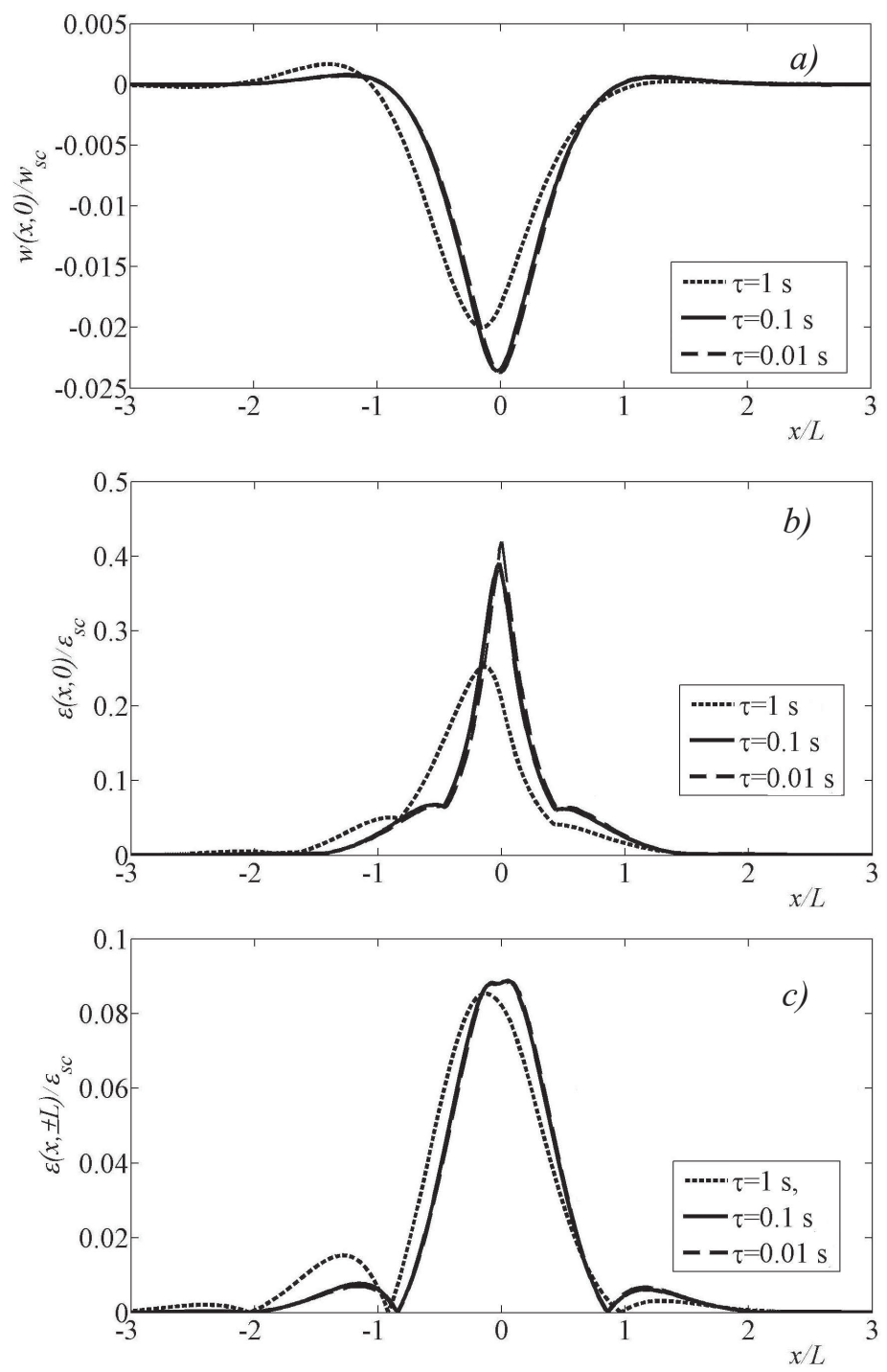

Figure 7: The deflections $(a)$ and strains $(b, c)$ for the load moving with the subcritical speed, $U=3 \mathrm{~m} / \mathrm{s}$, and different values of the retardation time. Here $L=10 \mathrm{~m}, h_{i}=10 \mathrm{~cm}$ and $H=2 \mathrm{~m}$.

The effect of the retardation time $\tau$ on the deflections and strains in the ice plate is demonstrated in Figures 7 and 8 . The calculations are performed for subcritical, $U=3 \mathrm{~m} / \mathrm{s}$ (Figure 7 ), and supercritical, $U=7 \mathrm{~m} / \mathrm{s}$ (Figure 8), speeds. Note that $\tau=0.1 \mathrm{~s}$ in all other figures of this paper. The decrease of the retardation time increases the maximum strains. However, the place where the ice can be broken, on the wall or along the centre line of the channel, is independent of $\tau$. For the subcritical speed the increase of the retardation time moves the points of the maximum strains and deflections from the center of the load (Figure $7 b, c)$. This lag of the position of maximum depression was measured in [32] and was used to estimate the viscoelastic characteristics of the ice. This lag was not detected for the supercritical speed (Figure 8), where the maximum strain does not change its position with $\tau$. The decrease of the retardation time extends 
the length of the interval of the significant deflections for the supercritical speed. The wave length of the ice plate oscillations in front of the load (Figure 8) is weakly dependent on $\tau$ and is equal to the wave length of the first hydroelastic wave with the phase speed $7 \mathrm{~m} / \mathrm{s}$. The oscillations with the same wave length but much smaller amplitude exist also behind the load, they are visible only for small $\tau$ (Figure 8 b,c). For the subcritical speed and $\tau<0.1 \mathrm{~s}$ the deflections and strains are weakly dependent on $\tau$.
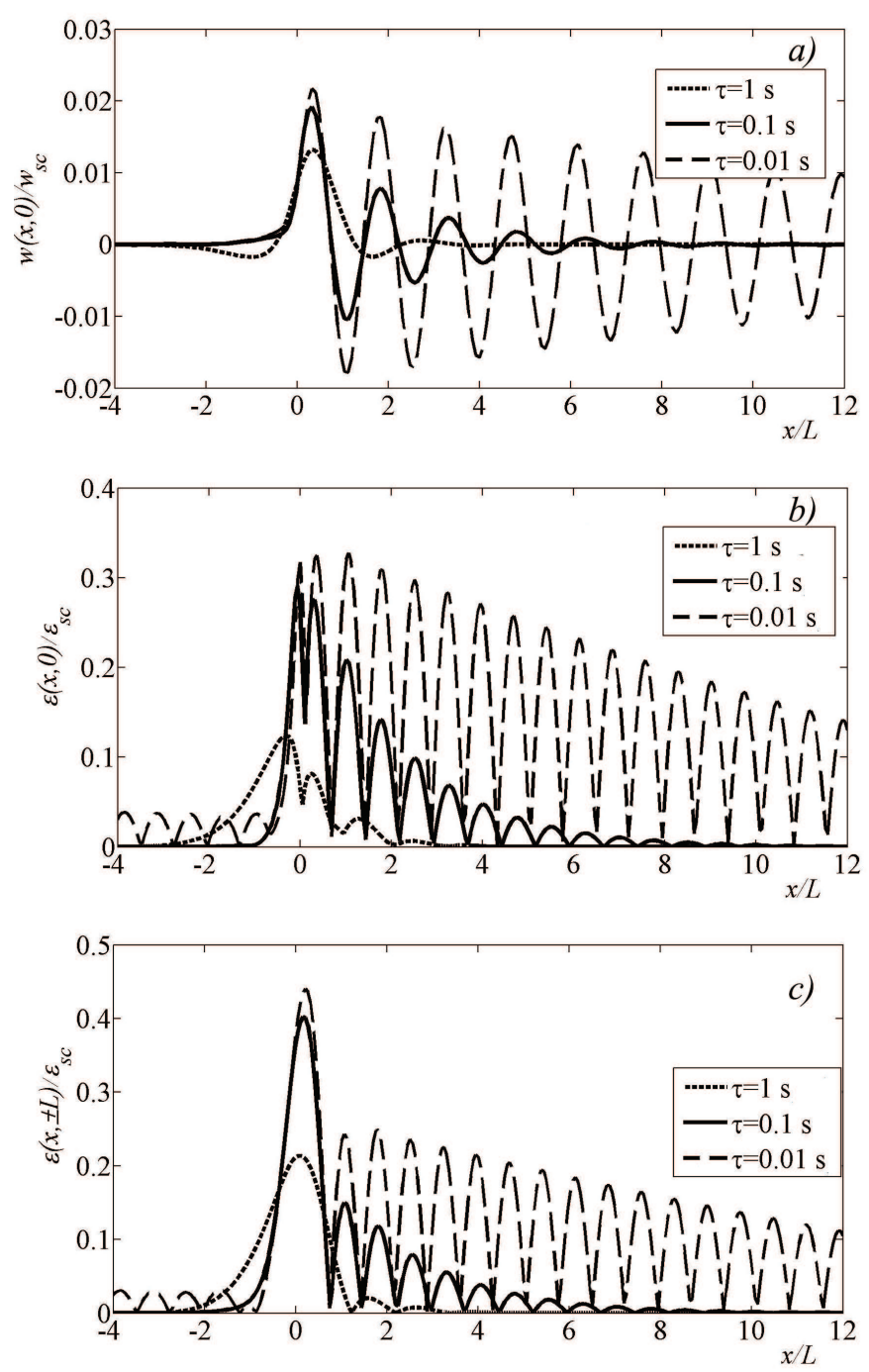

Figure 8: The deflections $(a)$ and strains $(b, c)$ for the load moving with the supercritical speed, $U=7 \mathrm{~m} / \mathrm{s}$, and different values of the retardation time. Here $L=10 \mathrm{~m}, h_{i}=10 \mathrm{~cm}$ and $H=2 \mathrm{~m}$. 

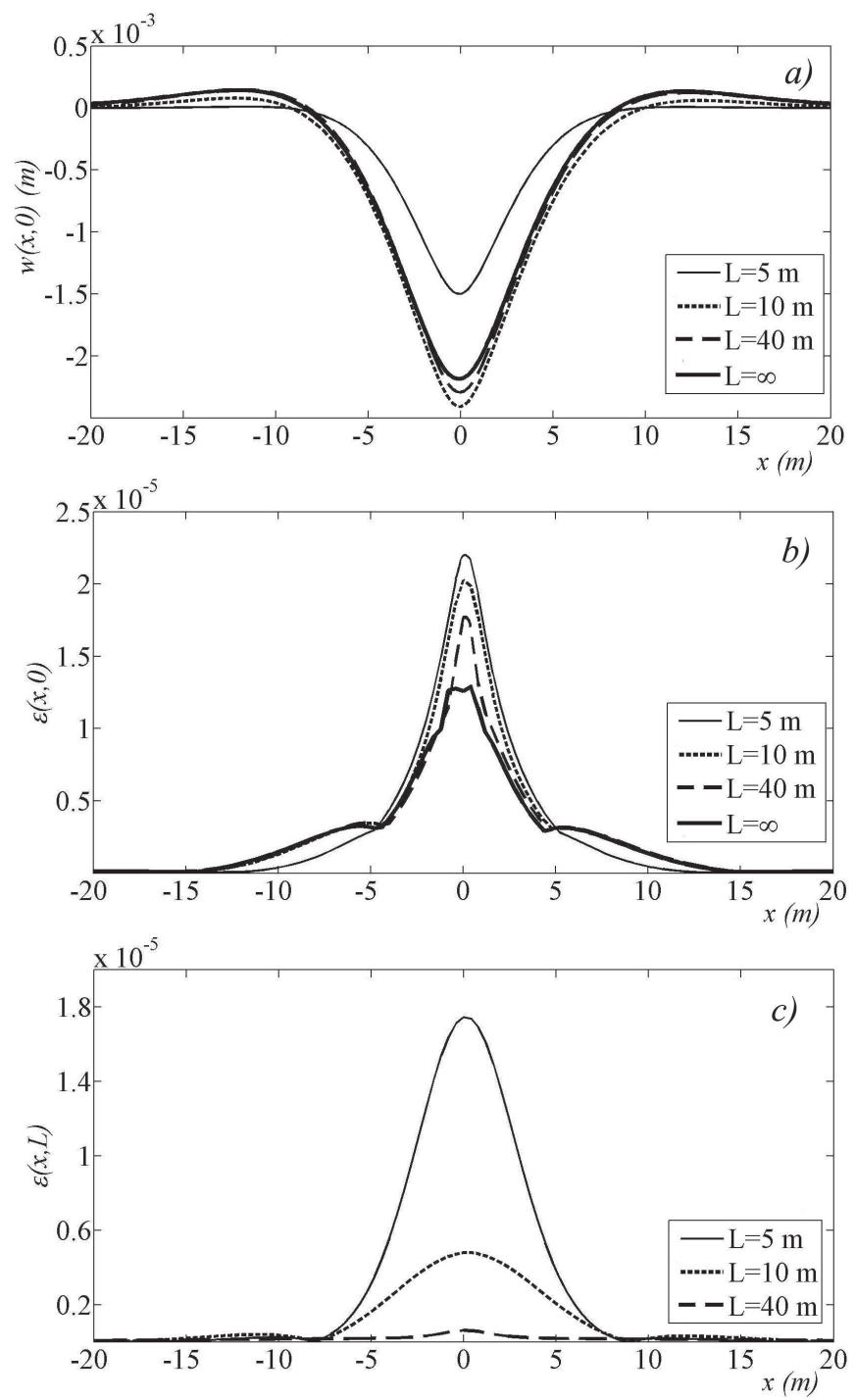

Figure 9: The dimensional deflections at the central line of the channel, $(a)$, and the physical strains at the central line of the channel $(b)$ and at the wall $(c)$ in the ice sheet for the subcritical speed $U=3 \mathrm{~m} / \mathrm{s}$ for different width of the channel. Here $h_{i}=10 \mathrm{~cm}, H=2 \mathrm{~m}$ and $L=5 \mathrm{~m}$ (thin solid line), $L=10 \mathrm{~m}$ (dotted line), $L=40 \mathrm{~m}$ (dashed line) and for the infinite ice plate (thick solid line).

The effect of the channel width $2 L$ on the deflections and strains in the ice sheet is demonstrated in Figures 9 and 10 for the subcritical speed $U=3 \mathrm{~m} / \mathrm{s}$ and the supercritical speed $U=7 \mathrm{~m} / \mathrm{s}$ of the load. The results of the calculations are presented in the dimensional variables for $P_{0}=1 \mathrm{kPa}$. Note that the critical speeds of the hydroelastic waves depend on the width of the channel (see [28]). These speeds increase with decrease of the channel width. The speed $U=7 \mathrm{~m} / \mathrm{s}$ is subcritical for $L=5 \mathrm{~m}$ but supercritical for other values of $L$ in Figure 10. This fact explains why the curves for $L=5 \mathrm{~m}$ are so different from all other curves in Figure 10. It is seen that both the deflections and strains approach their distributions for infinite ice plate given by (21), as the width of the channel increases. The deflections increase and the strains decrease with increasing the distance between the walls. 

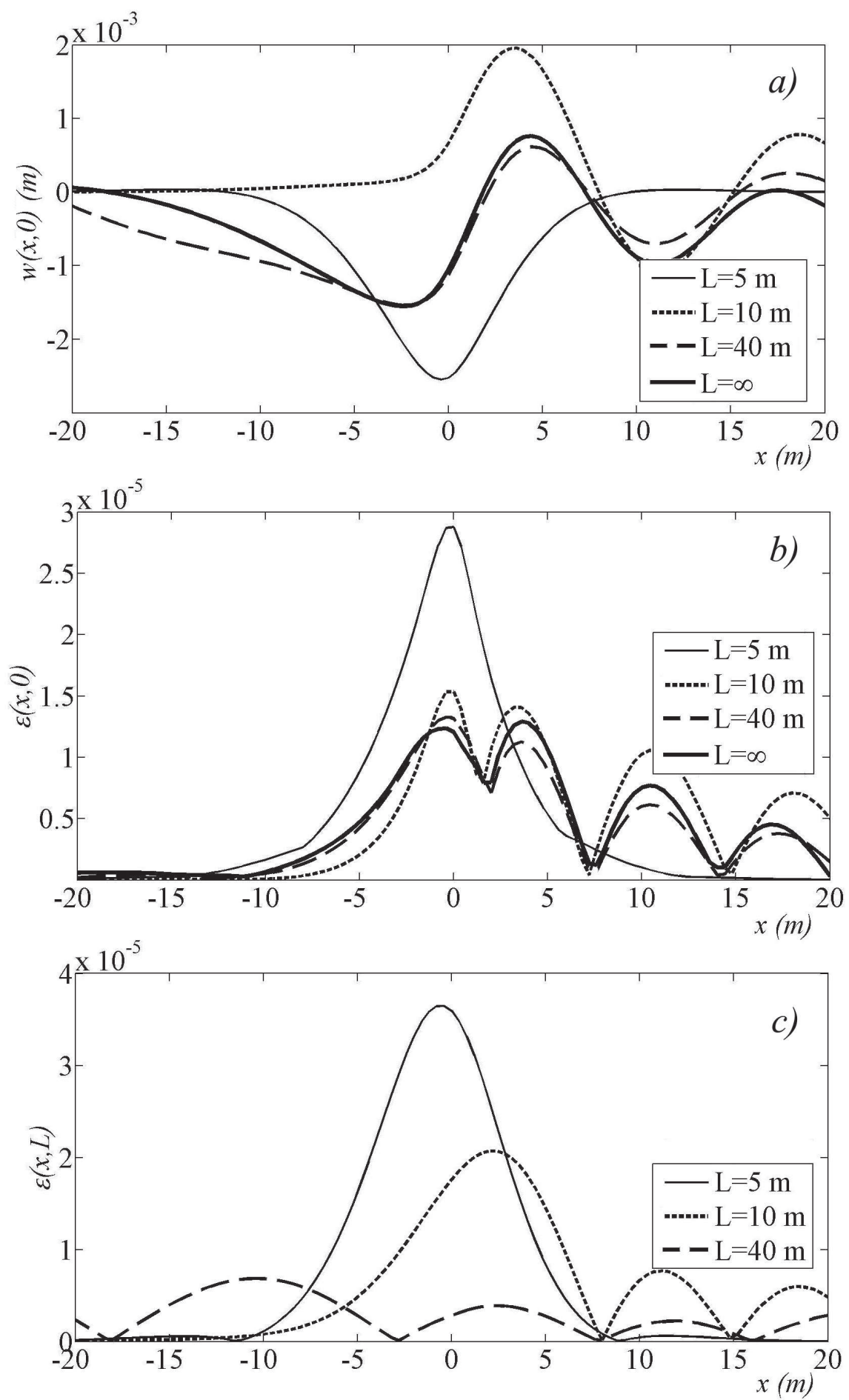

Figure 10: The dimensional deflections at the central line of the channel, $(a)$, and the physical strains at the central line of the channel $(b)$ and at the wall $(c)$ in the ice sheet for the supercritical speed $U=7 \mathrm{~m} / \mathrm{s}$ for different width of the channel.. Here $h_{i}=10 \mathrm{~cm}, H=2 \mathrm{~m}$ and $L=5 \mathrm{~m}$ (thin solid line), $L=10 \mathrm{~m}$ (dotted line), $L=40 \mathrm{~m}$ (dashed line) and for the infinite ice plate (thick solid line). 


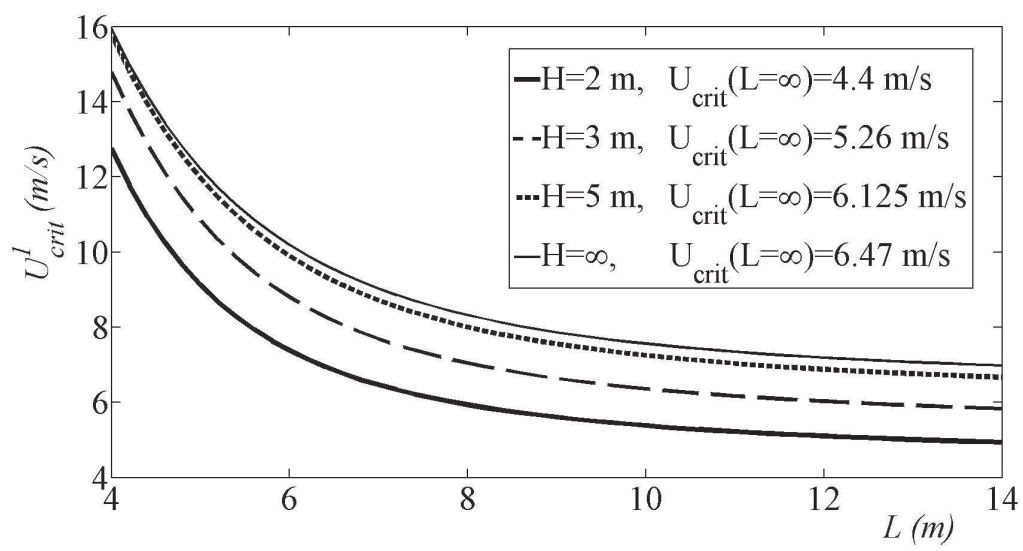

Figure 11: The critical speed of the first hydroelastic wave, $U_{\text {crit }}^{1}$, as a function of the channel width for different values of the channel depth, $H$. The critical speeds, $U_{\text {crit }}$, for the infinite ice plate and the corresponding depth of the channel are also shown. Here $h_{i}=10 \mathrm{~cm}$.

The effects of the channel depth and width on the critical speed of the first hydrodynamic wave are shown in Figure 11. These critical speeds are computed using the dispersion relations derived in [28]. The critical speed increases up to the speed value for the channel of infinite depth with increase of the channel depth. The critical speed decreases with the increase of the distance between the walls. Note that the characteristic length $\left(D / \rho_{l} g\right)^{1 / 4}$ of this ice plate is $2.48 \mathrm{~m}$. The effect of the channel width on the deflection and stresses in the ice sheet (Figures 9,10) and on the value of the lowest critical speed (Figure 11) is significant even for the channel width being much greater than the characteristic length of the ice sheet.

\section{Simplified models of ice-fluid interaction}

The problem under consideration can be simplified by neglecting the hydrodynamic pressure $p(x, y, 0, t)$ in the equation of the ice plate deflection (1), or by neglecting the dynamic component, $-\rho_{l} \varphi_{t}$, in the linearised Bernoulli equation (3). The first simplified model corresponds to a dry ice plate clamped to the vertical walls without water beneath the plate. It will be referred to as the Dry-Plate Model (DPM). This model can provide a reasonable approximation of the plate response for very thick ice covers or narrow channels. In the second simplified model, only the hydrostatic component, $-\rho_{l} g w$, of the liquid pressure is taken into account. This model is referred to as the HydroStatic Model (HSM). This model can be acceptable for very small speeds of the external load moving along the channel, when the dynamic component of the liquid pressure can be neglected. The ice response in both simplified models is described by the equations (1), (2), (4) and (5), where the pressure $p(x, y, 0, t)$ in (1) is given by $p=0$ for DPM and $p=-\rho_{l} g w(x, y, t)$ for HSM. The original fully coupled HydroDynamic Model (1) - (5) is referred to as HDM below. The corresponding simplified problems are solved by the method of section 3, with the matrix equation (17) being simplified to

$$
\begin{gathered}
\text { DPM : } \quad\left(-\alpha h \operatorname{Fr}^{2} \xi^{2}\right) \mathbf{I} \vec{a}+\beta(1-i \xi \varepsilon) \mathbf{Q} \vec{a}=-\vec{P}, \\
\text { HSM : } \quad\left(1-\alpha h \operatorname{Fr}^{2} \xi^{2}\right) \mathbf{I} \vec{a}+\beta(1-i \xi \varepsilon) \mathbf{Q} \vec{a}=-\vec{P} .
\end{gathered}
$$


The added-mass matrix $\mathbf{M}$ does not appear in both simplified models, which significantly simplifies computations. Comparing the solutions obtained by using the simplified equations (22) and (23) with the solution based on the complete equation (17) in terms of the plate deflection and stresses in the ice plate for the same conditions, we can conclude about importance of the hydrodynamic pressure and its dynamic component on the ice response. The approximate models DPM and HSM are also compared with the complete model in terms of the matrices of the systems (22), (23) and (17). Setting $\varepsilon=0$ and equating the determinants of these matrices to zero, we arrive at the corresponding dispersion relations which can be interpreted in terms of the frequency $\omega$ and the wave number $k$ of a hydroelastic wave propagating along the channel. Calculating the phase and group velocities of these waves, we obtain the critical speeds of the moving load within the original and simplified models. The analysis of the previous sections show that the critical speeds of the waves in the ice sheet are important for predicting response of the ice sheet to moving loads.

The problem of periodic hydroelastic waves propagating along a channel covered with an ice sheet is considered in the non-dimensional variables. The wave amplitude $A$ is taken as the deflection scale, the product $A L \omega$ is taken as the scale of the velocity potential, where $L$ is the half-width of the channel and $\omega$ is the wave frequency. The time scale is $1 / \omega$ and the length scale is $L$. The propagating waves are described by equations (1) - (5), where $\tau=0, P=0$ and

$$
w(x, y, t)=\operatorname{Re}\left[F(y) e^{i(\kappa x-t)}\right], \quad \varphi(x, y, z, t)=\operatorname{Re}\left[i \Phi(y, z) e^{i(\kappa x-t)}\right],
$$

see [28]. Here $k=\kappa / L$ is the corresponding wave number and $\Phi$ is the complex-valued potential which satisfies the Helmholz equation

$$
\Phi_{y y}+\Phi_{z z}=\kappa^{2} \Phi \quad(-1<y<1,-h<z<0)
$$

and the boundary conditions (4). Substituting (24) in (1), we obtain the equation

$$
-\alpha \gamma F+\beta\left[\kappa^{4}-2 \kappa^{2} F^{\prime \prime}+F^{\prime \prime \prime \prime}\right]=\gamma \Phi(y, 0)-F(y),
$$

with respect to the wave profile across the channel, $F(y)$, where $\gamma=L \omega^{2} / g$.

The solution of the equation (25) is obtained by the method of section 3 with $F(y)$ represented by series (13) but without using the Fourier transform. The corresponding matrix equation reads

$$
\left(\mathbf{T}-2 \kappa^{2} \mathbf{C}-\frac{\gamma}{\beta} \mathbf{P}^{l}\right) \vec{a}=0
$$

where $\vec{a}=\left(a_{1}, a_{2}, \ldots\right)^{T}, \mathbf{T}$ is the diagonal matrix with the elements $T_{j j}=\left\{\kappa^{4}+\lambda_{j}^{4}-\gamma \alpha / \beta\right\}_{j=1}^{\infty}$, the matrix $\mathbf{C}$ and the eigen-values $\lambda_{j}$ are defined is section 3. The matrix $\mathbf{P}^{l}$ in (26) is zero for the model DPM, is diagonal, $\mathbf{P}^{l}=-1 / \gamma \mathbf{I}$, for the model HSM, and includes the added-mass matrix $\mathbf{M}$ for the complete model HDM, $\quad \mathbf{P}^{l}=\mathbf{M}-1 / \gamma \mathbf{I}$. The dispersion relations $\omega_{n}(k)$, where $\omega_{1}(k)<\omega_{2}(k)<\omega_{3}(k)<\ldots$, are obtained as the roots of the determinant of the matrix in (26). This matrix multiplied by $\beta$ is equal to the matrix of the system (17) and to the corresponding matrices in (22), (23) for the simplified models if we set $\gamma=h \mathrm{Fr}^{2} \xi^{2}$ and $\kappa=\xi$ in (26). The latter equalities lead to the relation $\omega(k)=U k$ in the dimensional variables. Therefore, 
the determinant of the matrix $\mathbf{A}$ in system (17) with $\tau=0$ is equal to zero at such $\xi$ that the speed of the load $U$ is equal to the corresponding phase speed $c^{(n)}(\xi)=\omega_{n}(\xi) / \xi$ for a certain n. Correspondingly, the critical speeds of the load, $U_{c r i t}^{(n)}$, are equal to the minimum values of the phase speeds $c^{(n)}(\xi)$. Note that the minimum values of the phase speeds are equal to the corresponding values of the group speeds, $c_{g}^{(n)}\left(\xi_{\text {crit }}^{(n)}\right)=\left(d \omega_{n} / d \xi\right)\left(\xi_{\text {crit }}^{(n)}\right)$, where $c^{(n)}\left(\xi_{\text {crit }}^{(n)}\right)=\min _{\xi \geq 0}\left[c^{(n)}(\xi)\right]$

The dispersion relations for the complete model HDM were studied in [28]. In this section, we start with the dispersion relations for the models DPM and HSM, and compare the obtained results, $\omega_{n}^{\mathrm{DPM}}(k)$ and $\omega_{n}^{\mathrm{HSM}}(k)$, with the relations $\omega_{n}^{\mathrm{HDM}}(k)$ from [28].

The numerical analysis of this section aims to investigate the effects of the components of the liquid pressure on the hydroelastic waves. The numerical study is performed for the same values of the characteristics of the ice plate, channel and moving load specified in section 4, unless other values of these characteristics are specified.

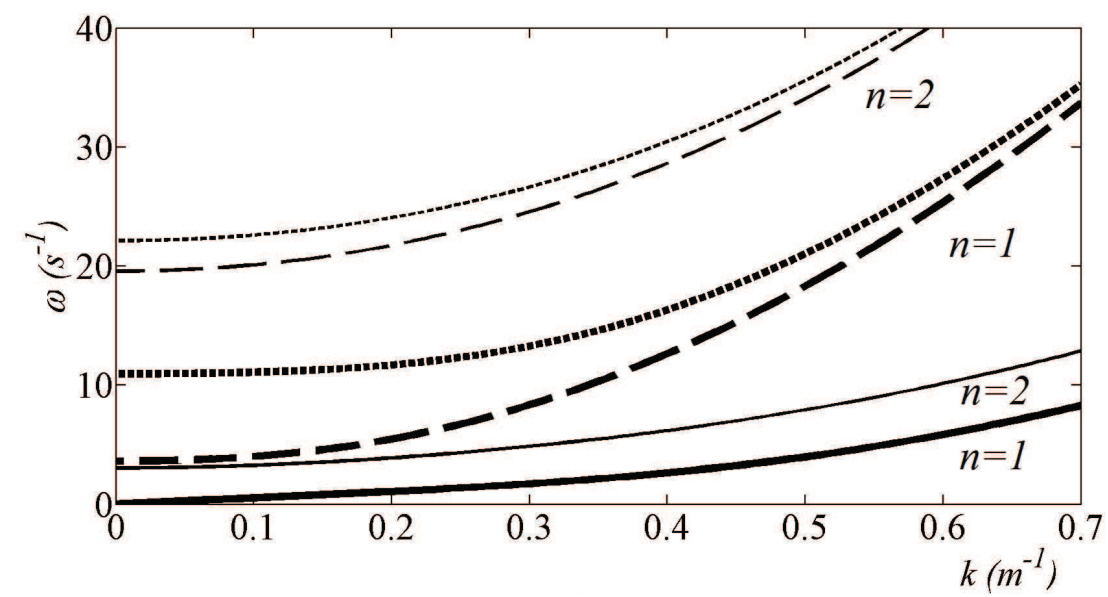

Figure 12: The dispersion relations of the first hydroelastic wave (thick lines) and the second hydroelastic wave (thin lines) in the models HDM (solid line), DPM (dashed line) and HSM (dotted line). Here $L=10 \mathrm{~m}, h_{i}=10 \mathrm{~cm}$ and $H=2 \mathrm{~m}$.

The hydroelastic propagating waves in the model HDM exist for any frequency $\omega$ because $\omega_{1}(0)=0$. However, these waves exist only for $\omega>3.82 \mathrm{sec}^{-1}$ in DPM and for $\omega>10.95 \mathrm{sec}^{-1}$ in HSM. For any constant frequency $\omega>10.95 \mathrm{sec}^{-1}$ the wave length, $\lambda=2 \pi / k$, satisfies the equalities $\lambda_{1}^{\mathrm{HSM}}<\lambda_{1}^{\mathrm{DPM}}<\lambda_{1}^{\mathrm{HDM}}$ where the index 1 stand for the first hydroelastic wave. Note that the ice cover can be modelled as an elastic plate only for waves which are much longer than the ice thickness $h_{i}$, say $\lambda>10 h_{i}$. This restriction yields $k<\pi /\left(5 h_{i}\right)$, which gives $k<6.5 \mathrm{~m}^{-1}$ for $h_{i}=10 \mathrm{~cm}$ and $k<0.65 \mathrm{~m}^{-1}$ for $h_{i}=1 \mathrm{~m}$. The Figure 12 shows that the dynamic interaction between the ice cover and the liquid in the channel makes the hydroelastic waves longer and permits waves with any frequencies, in contact to the simplified models DPM and HSM. 


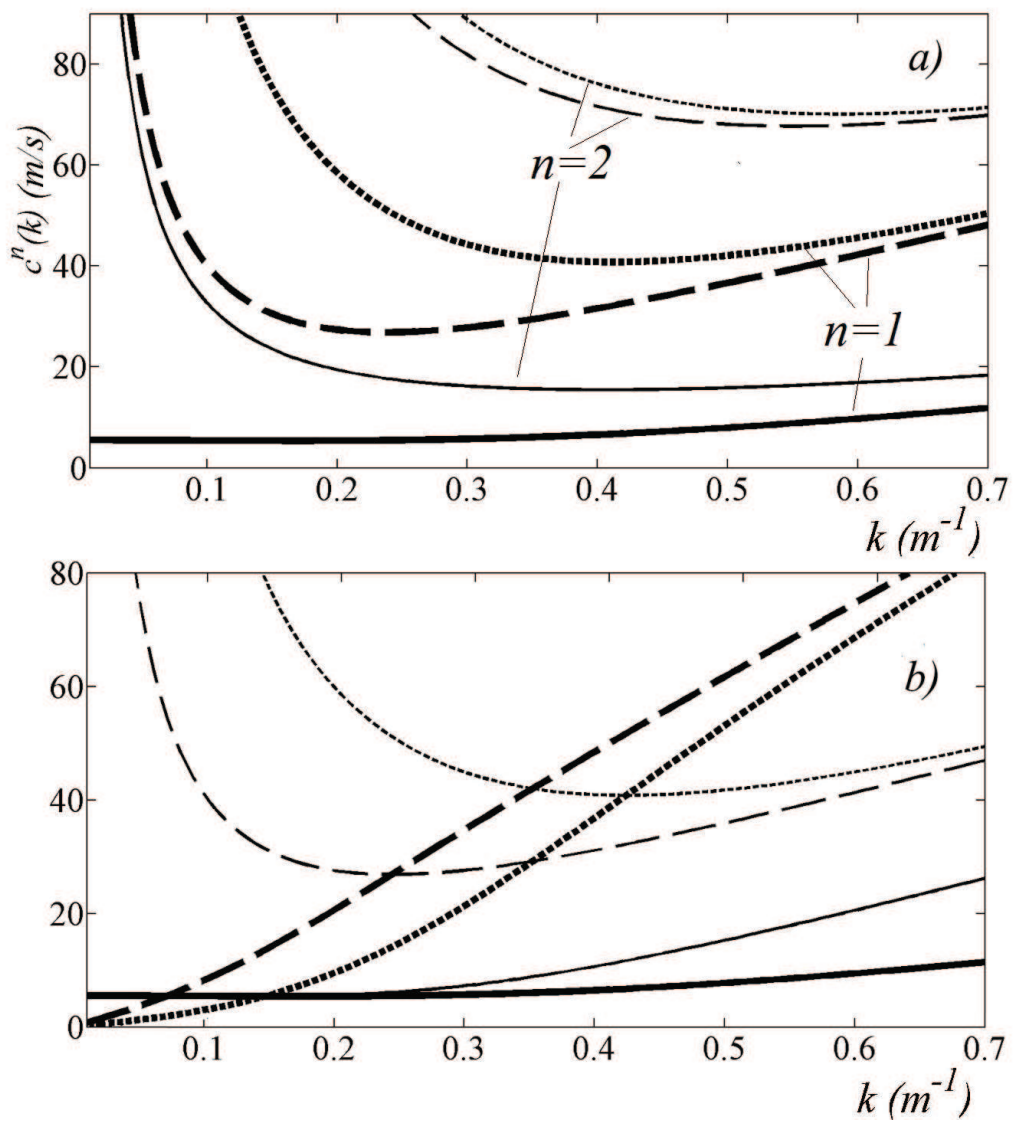

Figure 13: (a) The phase speeds (in $\mathrm{m} / \mathrm{s}$ ) of the first (thick line) and second (thin line) hydroelastic waves for the models HDM (solid line), DPM (dashed line) and HSM (dotted line). (b) The phase (thin lines) and group (thick lines) speeds of the first hydroelastic wave as predicted by the three models. Here $h_{i}=10 \mathrm{~cm}, H=2 \mathrm{~m}$ and $L=5 \mathrm{~m}$.

The phase speeds, $c^{(n)}(k)=\omega_{n}(k) / k$, are shown in Figure $13 a$ for the first $(n=1)$ and second $(n=2)$ waves propagating along the frozen channel. The phase speeds predicted by the complete model HDM are smaller than the phase speeds in the model DPM, which in turn are smaller than the corresponding speeds in the model HSM. The hydrodynamic component of the liquid pressure significantly affects the phase speed of the first hydroelastic wave for small wave numbers. A finite value of the phase speed as $k \rightarrow 0$ exists only for the complete model HDM. The curves of the phase speeds have well-defined minima, where the group speeds and the phase speeds are equal (see Figure 13b). The minima determine the critical speeds for the hydroelastic waves within the models. The critical speeds of the first hydroelastic wave are $U_{\text {crit }}^{(1)}=5.38$ $\mathrm{m} / \mathrm{s}$ in the model HDM, $U_{\text {crit }}^{(1)}=26.86 \mathrm{~m} / \mathrm{s}$ in the model DPM and $U_{\text {crit }}^{(1)}=40.79 \mathrm{~m} / \mathrm{s}$ in the model HSM. Note that the critical speed in the model HSM is about 7.5 times higher than the critical speed in the complete model HDM and is about 1.5 times higher than the critical speed of the "dry" plate. Therefore, both simplified models significantly overpredict the critical speeds. Figure $13 b$ shows that the group speed of the first hydroelastic wave is very close to the phase speed in HDM for long waves $\left(k \leq 0.2 \mathrm{~m}^{-1}\right)$ and is approximately equal to the critical speed $5.38 \mathrm{~m} / \mathrm{s}$. This result is not valid for the simplified models which do not account for the liquid dynamics. 
The computations performed with a low number of terms in the series (13) show that the single-mode approximation for the first hydroelastic wave and the two-mode approximation for the second hydroelastic wave in the complete model HDM provide the dispersion relations, group and phase speeds which well approximate the results obtained with large numbers of modes, $N_{\text {mod }}=15$, in (13). The single-mode approximation and the two-mode approximation of the dispersion relations are computed by using the following equations

$$
\begin{gathered}
\kappa^{4}+\lambda_{1}^{4}-\gamma \alpha / \beta-2 \kappa^{2} C_{11}-\frac{\gamma}{\beta} P_{1}^{l}=0 \\
\operatorname{det}\left[\left(\begin{array}{cc}
\kappa^{4}+\lambda_{1}^{4}-\gamma \alpha / \beta & 0 \\
0 & \kappa^{4}+\lambda_{2}^{4}-\gamma \alpha / \beta
\end{array}\right)-2 \kappa^{2}\left(\begin{array}{cc}
C_{11} & C_{12} \\
C_{21} & C_{22}
\end{array}\right)-\right. \\
\left.-\frac{\gamma}{\beta}\left(\begin{array}{cc}
P_{11}^{l} & P_{12}^{l} \\
P_{21}^{l} & P_{22}^{l}
\end{array}\right)\right]=0
\end{gathered}
$$

These equations are obtained from (26) with only one (first) mode in the series (13) for the single-mode approximation (26) and with the first two modes in the series (13) for the two-mode approximation. Note that the approximate values of $\gamma$ and consequently $\omega_{1}(k)$ are given explicitly by $(27)$. To find $\omega_{2}(k)$, we need to solve the quadratic equation (28). Equation (27) provides that $\omega_{1}^{\mathrm{HSM}}(k)>\omega_{1}^{\mathrm{DPM}}(k)$, which agrees with Figure 12 , and $U_{c r i t, \mathrm{HSM}}^{(1)}$ being greater than $U_{\text {crit,DPM }}^{(1)}$, as a result of this inequality. 

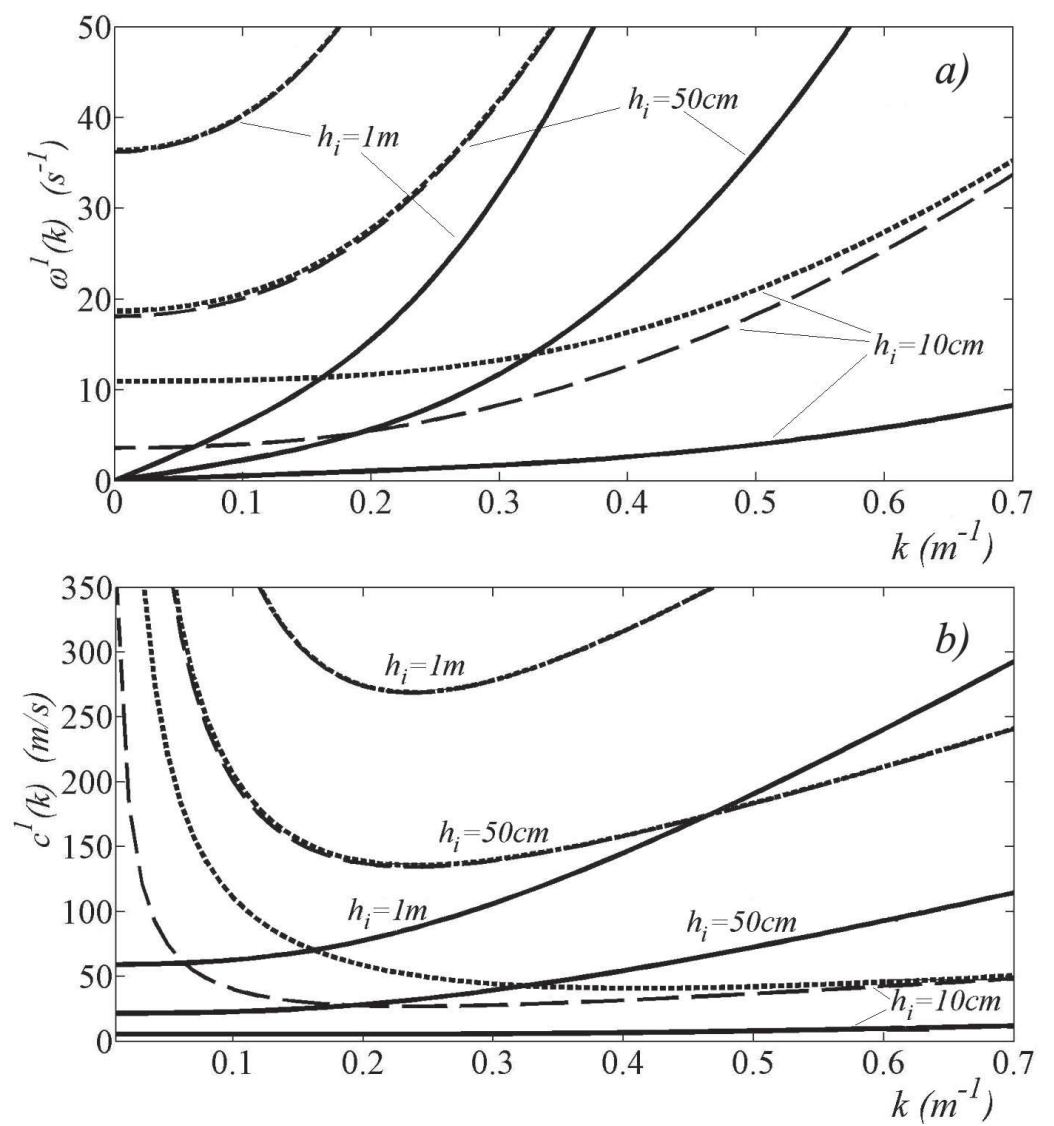

Figure 14: The dispersion relations (a) and the phase speeds (b) of the first hydroelastic wave in the models HDM (solid line), DPM (dashed line) and HSM (dotted line). Here $H=2 \mathrm{~m}, L=5 \mathrm{~m}$ and $h_{i}=10 \mathrm{~cm}, 50 \mathrm{~cm}$ and $100 \mathrm{~cm}$.

One may expect that the simplified models approximate better the dispersion relations and the phase speeds of the hydroelastic waves in the channel for thicker ice plates. Figure 14 shows that this is not the case. The dispersion relations and phase speeds of the first hydroelastic wave in the models HDM, HSM and DPM for different thicknesses of the ice cover, $h_{i}$, are shown in Figure 14 . The ice thickness varies from $10 \mathrm{~cm}$ to $100 \mathrm{~cm}$. The frequency $\omega_{1}(k)$ increases with increase of the ice thickness for these models. The Figure 14 demonstrates that the dynamic component of the liquid pressure is an important factor in predicting hydroelastic waves in the ice cover for any thickness of it. The frequencies and the phase speeds predicted by HSM and DPM indeed approach each other with increasing the ice plate thickness. However the difference between the predictions by the complete model HDM and the simplified models increases with increase of the ice thickness for long waves.

The convergence of the wave frequency of the first wave, $\omega^{1}(k)$, as the length of the wave decreases, $k \rightarrow \infty$, is shown in Figure 15 for the channel of width $10 \mathrm{~m}$ (dashed lines) and the infinite ice plate (solid lines) for different thicknesses of the ice sheet. The dispersion relations for the infinite plate are given by (see [1]) 

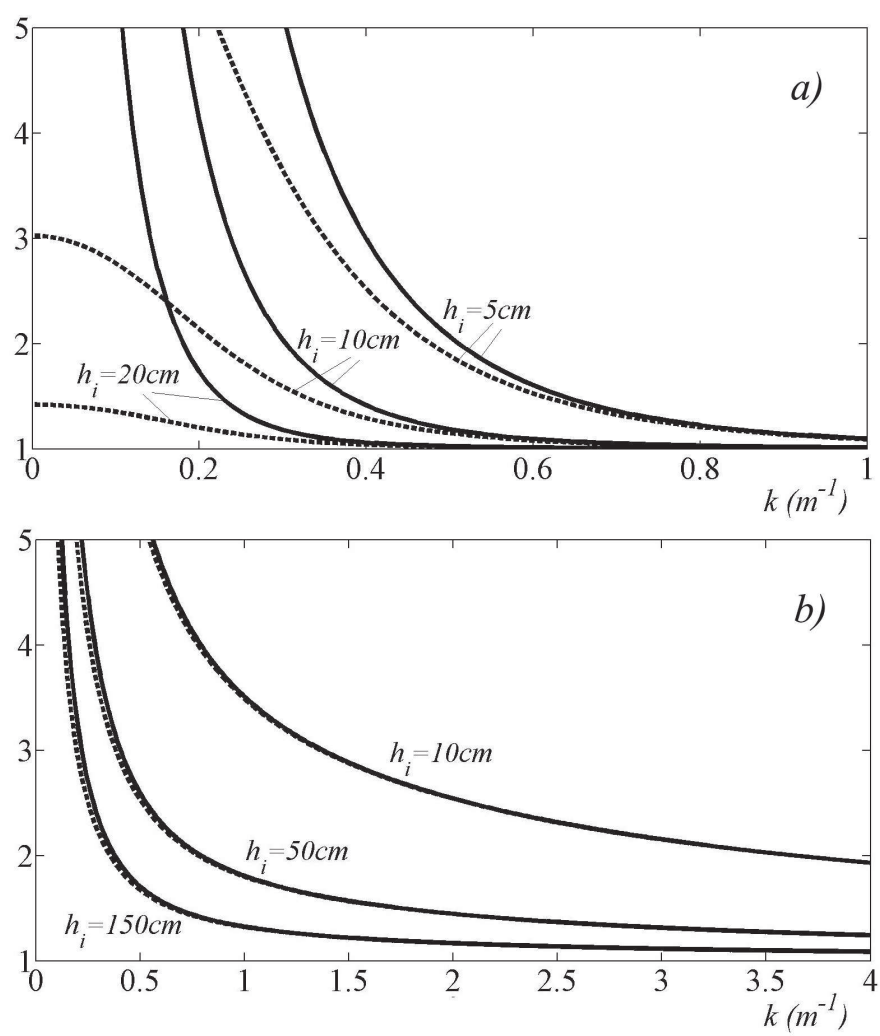

Figure 15: The ratio $\omega_{\mathrm{HSM}}^{1} / \omega_{\mathrm{DPM}}^{1}$ of the wave frequency in the HSM to the wave frequency in the DPM (a), and the ratio $\omega_{\mathrm{HSM}}^{1} / \omega_{\mathrm{HDM}}^{1}$ of the wave frequency in the HSM to the wave frequency in the HDM $(b)$ as functions of the wave number for the channel (dashed line) and for the infinite ice plate (solid lines). Here $H=2 \mathrm{~m}, L=5 \mathrm{~m}$ and $h_{i}=10 \mathrm{~cm}, 50 \mathrm{~cm}$ and $100 \mathrm{~cm}$.

$$
\omega_{\mathrm{DPM}}(k)=\sqrt{\frac{D k^{4}}{\rho_{i} h_{i}}}, \quad \omega_{\mathrm{HSM}}(k)=\sqrt{\frac{D k^{4}+\rho_{l} g}{\rho_{i} h_{i}}}, \quad \omega_{\mathrm{HDM}}(k)=\sqrt{\frac{D k^{4}+\rho_{l} g}{\rho_{i} h_{i}+\rho_{l} / k \tanh (k H)}} .
$$

The dispersion relation $\omega_{\mathrm{HDM}}(k)$ can be approximated by the dispersion relation $\omega_{\mathrm{HSM}}(k)$ if $k h_{i} \gg 1$, which gives $h_{i} / \lambda \gg 1 / 2 \pi$, where $\lambda$ is the wave length. However, the thin plate model of ice cover can be used only for wave length $\lambda$ much larger than the ice thickness, $h_{i}$, say, for $\lambda>10 h_{i}$. The inequality $h_{i} / \lambda \gg 1 / 2 \pi$ does not hold for such long hydroelastic waves. Therefore, $\omega_{\mathrm{HSM}}(k)$ cannot approximate $\omega_{\mathrm{HDM}}(k)$ within the thin plate model of ice. This is a consequence of the fact that the densities of the ice and water are close to each other. It is interesting that $\omega_{\mathrm{HSM}}(k)$ can be approximated by $\omega_{\mathrm{DPM}}(k)$, which does not account for the presence of the liquid beneath the ice sheet, if $\rho_{l} g / D k^{4} \ll 1$. Here $\left(D / \rho_{l} g\right)^{1 / 4}$ is the characteristic length of the ice sheet, $l_{c}$. Then the latter inequality yields $\lambda / l_{c} \ll 2 \pi$. This implies that the presence of the liquid can be neglected for very short waves. We conclude that the hydrodynamic pressure strongly affects the characteristics of hydroelastic waves in thin ice covers. 

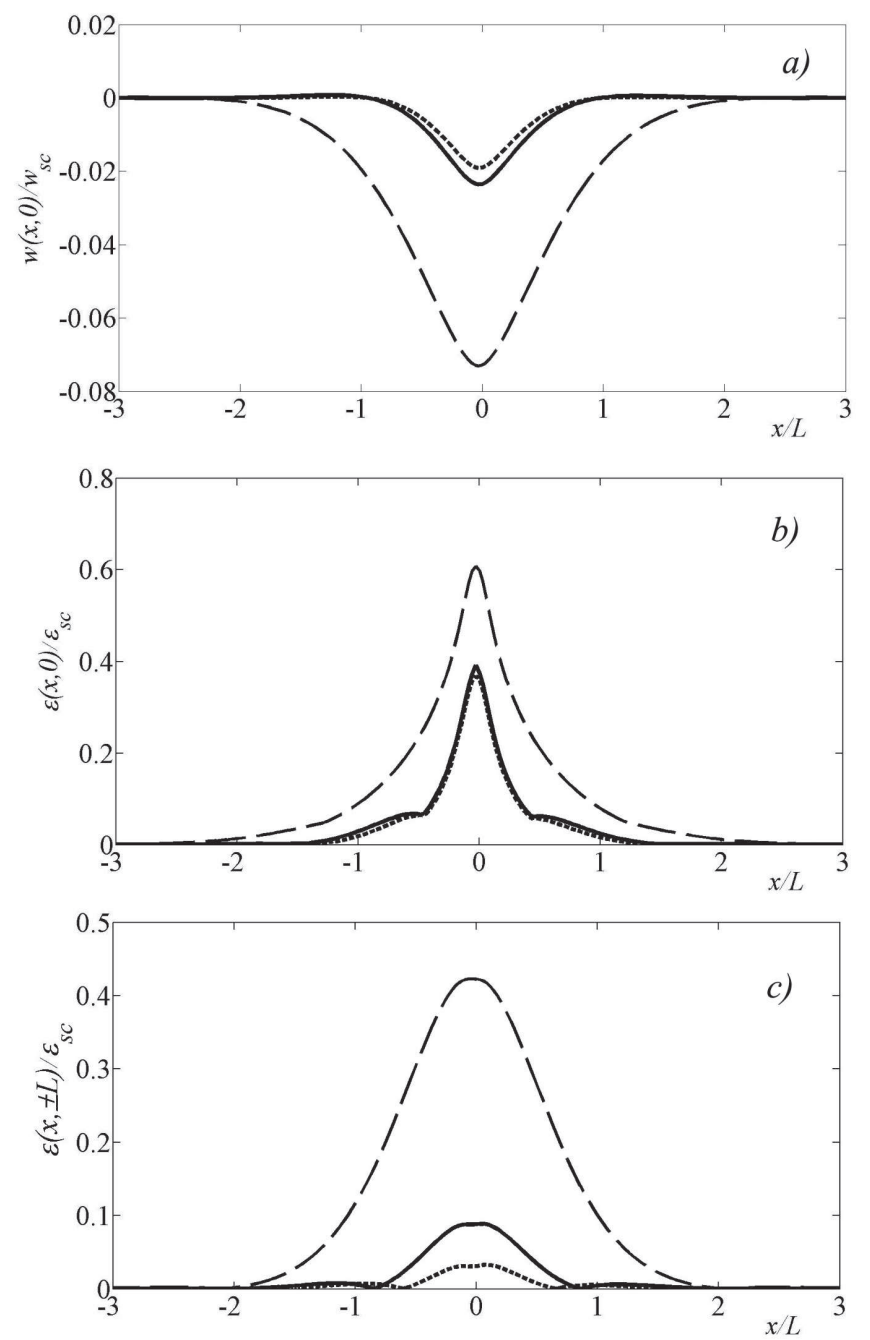

Figure 16: The deflections (a), the strains along the centre line of the channel (b) and the strains along the wall (c) predicted by the models HDM (solid lines), DPM (dashed line) and HSM (dotted line) for the subcritical speed $U=3 \mathrm{~m} / \mathrm{s}$. Here $H=2 \mathrm{~m}$, $L=5 \mathrm{~m}$ and $h_{i}=10 \mathrm{~cm}$.

However, the simplified models can be used for the analysis of ice response caused by a moving load. The deflections and strains predicted by the complete and simplified models are shown in Figure 16 and 17 for the load moving along the channel at the speeds $3 \mathrm{~m} / \mathrm{s}$ and $7 \mathrm{~m} / \mathrm{s}$ correspondingly. Figure 16 demonstrates that the deflection of the ice plate and the strains on the central line of the channel are well approximated by the HSM model for the subcritical speed of the load. However, the model HSM underpredicts the strains at the walls. The model DPM overpredicts both the deflections and strains for the subcritical and supercritical speeds of the load. We can argue that the simplest model DPM, which does not account for the presence of the liquid beneath the ice cover can be used to derive rough estimates of the stresses and deflections caused by a moving load. The accurate prediction of the ice plate response requires the complete model HDM. 

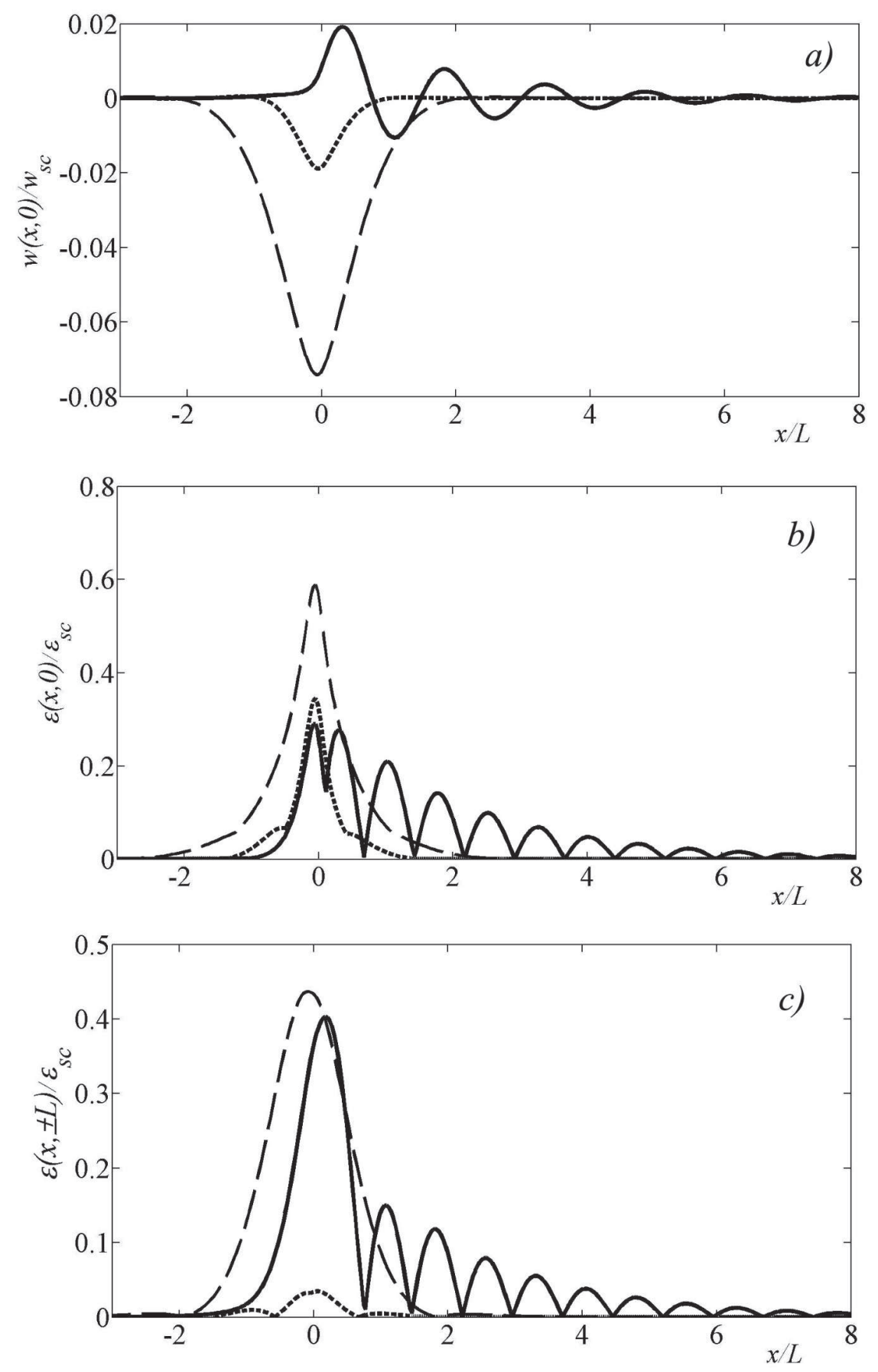

Figure 17: The deflections (a), the strains along the centre line of the channel (b) and the strains along the wall (c) predicted by the models HDM (solid lines), DPM (dashed line) and HSM (dotted line) for the subcritical for the model HDM speed $U=7 \mathrm{~m} / \mathrm{s}$. Here $H=2 \mathrm{~m}, L=5 \mathrm{~m}$ and $h_{i}=10 \mathrm{~cm}$. 


\section{Conclusion}

The effect of the vertical walls on the deflections and strains in the ice cover of a channel caused by a moving load has been studied. The characteristics of the ice cover, the depth of the channel and the parameters of the external load were not varied in the analysis. It was shown that the speed of the load strongly affects the response of the ice cover. There are many critical speeds of the load moving along the channel. The ice response is highest for the speed of the load close to the critical speed, $U_{c r i t}^{(1)}$, of the first hydroelastic wave propagating along the channel [28]. The presence of the walls of the channel is important even for wide channels where the width of the channel is much greater than the characteristic length of the ice cover. The presence of the walls increases the strains but decreases the deflections in comparison with the ice plate of infinite extent. The ice response is localised near the moving load for subcritical speeds of the load $U<U_{c r i t}^{(1)}$. For the speeds of the load between $U_{c r i t}^{(1)}$ and $U_{c r i t}^{(2)}$, the ice deflection oscillates in front of the load. These oscillations correspond to the first hydroelastic wave with the phase speed equal to the speed of the load. If the speed of the load is between $U_{c r i t}^{(2)}$ and $U_{c r i t}^{(3)}$, there are a long second hydroelastic wave behind the load and short first and second hydroelastic waves in front of the load.

The effects of the static and dynamic components of the liquid pressure on the dispersion relations, phase speeds of the wave propagating along the channel, and the deflections and strains caused by a moving load have been studied. The hydrodynamic pressure decreases the wave frequencies, phase and critical speeds of hydro-elastic waves. Simplified models of floating ice, which do not account for the dynamic component of the liquid pressure, are not recommended for analysis of hydroelastic waves. However, the deflections and strains of the ice plate caused by a moving load are well approximated by the deflections and strains of the ice plate calculated only with the hydrostatic pressure for the load speed smaller than the critical speed of the first hydroelastic wave. The simplest model of dry ice plate can be used for rough estimates of the stresses caused by a moving load.

The maximum strain is achieved on the central line of the channel for subcritical speeds, $U<U_{c r i t}^{(1)}$, but on the walls for critical and supercritical speeds, $U \geq U_{c r i t}^{(1)}$. The critical speeds, $U_{c r i t}^{(n)}$, depend on both the width and depth of the channel, increasing with the depth and decreasing with increase of the distance between the channel walls. This implies that for a vehicle moving along a frozen channel of slowly varying depth at a constant speed, the ice can be broken in the place of small depth even the transportation is safe in places of larger depth of the channel. Correspondingly, an aircraft landing on a frozen converging bay with decreasing speed can be dangerous if the speed of the aircraft becomes close to the local critical speed. For example, Figure 11 shows that landing on a converging bay of depth $2 \mathrm{~m}$ with thickness of ice cover $10 \mathrm{~cm}$ at speed $10 \mathrm{~m} / \mathrm{s}$ is safe far from the shore, where the bay is wide. However, the ice can be broken closer to the shore, where the distance between the bay banks is about $9 \mathrm{~m}$ and the local critical speed is close to the speed of the aircraft.

The ice model in the present study is simplified and does not account for several important properties of ice covers such as nonuniformity of the ice through its thickness and along the channel. It has been shown that the critical speeds increase with increase of the ice thickness (see Figure 14b). If the ice thickness decreases along the channel from $1 \mathrm{~m}$ to $0.5 \mathrm{~m}$ and a vehicle moves at speed $30 \mathrm{~m} / \mathrm{s}$, then the ice can be 
broken there, where the local critical speed is close to $30 \mathrm{~m} / \mathrm{s}$. Note that, in this case, the stresses are maximum at the walls but not under the vehicle (see Figure $4 b$ ). This means that the vehicle may pass the dangerous place in the channel safely because the ice will detach from the wall and the stresses in the ice plate will be released.

The maximum strains in the ice plate strongly depend on the retardation time $\tau$ and increase with decrease of the retardation time. This parameter describes how quickly disturbances decay in ice with the distance from a source and in time. The values of this parameter can be estimated using field measurements. The stresses in the ice cover are limited when a vehicle moves on the ice even at a critical speed. This is due to the viscoelastic properties of the ice and non-linear interaction between the ice cover and water in the channel. It has been found that the damping effects are of major importance when the speed of the vehicle is greater than the first critical speed, $U_{c r i t}^{(1)}$, in the channel. As to the first critical speed, both non-linearity and damping can be responsible for finite stresses when the vehicle speed is close to $U_{c r i t}^{(1)}$. To determine the conditions when the non-linear effects should be included in the model of ice response, the term in the plate equation (1) with $\tau$ is estimated as $O\left(D \tau U_{c r i t}^{(1)} w_{s c} / L^{5}\right)$ by using the scales of section 2 . The non-linear effects come, in particular, from the hydrodynamic pressure $p(x, y, w(x, y, t), t)$ in (1). The Bernoulli equation provides the estimate of non-linear terms in the Bernoulli equation, $O\left(\rho_{l} w_{t}^{2}\right)=O\left(\rho_{l}\left(U_{c r i t}^{(1)}\right)^{2} w_{s c}^{2} / L^{2}\right)$, where the deflection scale is $w_{s c}=P_{0} /\left(\rho_{l} g\right)$. The obtained estimates yield that the dissipation effect is dominant and non-linearity of the ice response in the channel can be neglected if

$$
\tau E h_{i}^{3} g \gg U_{c r i t}^{(1)} P_{0} L^{3}
$$

This inequality implies that the non-linear effects can be dominant for wide channels (large $L$ ), heavy weight of the moving vehicle (large $P_{0}$ ), deep channels (large $U_{c r i t}^{(1)}$ ), ice covers of small thickness $h_{i}$, ice with small Young's modulus $E$ and small retardation time $\tau$. For the conditions of Figure 4 with $P_{0}=210 \mathrm{~N} / \mathrm{m}^{2}$, which is higher than the limit $206 \mathrm{~N} / \mathrm{m}^{2}$ evaluated for these conditions, the non-linear effects provide the contribution to the maximum strain comparable to the contribution from the viscoelasticity of the ice, if the retardation time is smaller than $0.03 \mathrm{~s}$. Note that, in the experiments reported in [23], the retardation time of the ice in the ice tank was estimated in the range from 3 to 10 seconds. In summary, even if the non-linear effects are important for the infinite ice sheet, they could be negligible for the ice response to the same load but in a channel, where the viscoelastic effects may dominate.

The value of the retardation time determines whether the ice will break or not. Therefore we need to consider practically justified ranges of the ice parameters to draw a conclusion about ice fracture or yield. The approach of this paper makes it possible to incorporate in the analysis more realistic models of viscoelastic ice as that derived by Tabata [33] without difficulties.

Only symmetric, even in $y$ across the channel, deflections of the ice sheet were studied in this paper. Asymmetric, odd in $y$, responses of the ice cover between the vertical walls are investigated by the same method using odd solutions (modes) of the problem (14). Both even and odd propagating hydroelastic waves in a channel were studied in [28]. Figure $6 b$ in [28] gives the critical speed of the first odd hydroelastic wave about $9 \mathrm{~m} / \mathrm{s}$, which is in between the critical speed of first and second even hydroelastic waves, 


\section{Acknowledgments}

This work was partially supported by the grants RFBR 13-08-01097 "Ice-structure interaction with unknown contact zones" and 16-08-00291 "Hydroelastic and thermodynamic effects with interaction of poroelastic ice and structures". 


\section{References}

[1] Squire V, Hosking R, Kerr A, Langhorne P. Moving loads on ice plates. Kluwer Academic Publishers; 1996.

[2] Kheysin DYe. Moving load on an elastic plate which floats on the surface of an ideal fluid.(in Russian) Izv. Akad. Nauk SSSR, Otd. Tekh. Nauk, Mekh. i Mashinostroenie 1963 (1); 178-180.

[3] Kheysin DYe. Dynamics of floating ice covers. (in Russian) Gidrometeorologichoskoe Izdatelstvo, Leningrad, Technical Translation FSTC-HT-23-485-69. US Army Foreign Science and Technology Center 1967.

[4] Sabodash PF, Filippov IG. A dynamic problem for a thin elastic plate. International Applied Mechanics 1967; 3(6): 28-31.

[5] Nevel DE. Moving loads on a floating ice sheet. No. CRREL-RR-261. Cold regions research and engineering lab. Hanover NH, 1970.

[6] Părău EI. Dias F. Nonlinear effects in the response of a floating ice plate to a moving load. Journal of Fluid Mechanics 2002; 460: 281-305.

[7] Hegarty GM, Squire V. A boundary-integral method for the interaction of largeamplitude ocean waves with a compliant floating raft such as a sea-ice floe. Journal of Engineering Mathematics 2008;62(4):355-372.

[8] Bonnefoy F, Meylan MH, Ferrant P. Nonlinear higher-order spectral solution for a twodimensional moving load on ice. Journal of Fluid Mechanics 2009;621:215-242.

[9] Plotnikov PI, Toland JF. Modelling nonlinear hydroelastic waves. Philosophical Transactions of the Royal Society of London A: Mathematical, Physical and Engineering Sciences 2011;369.1947: 2942-2956.

[10] Guyenne P, Părău EI. Asymptotic Modeling and Numerical Simulation of Solitary Waves in a Floating Ice Sheet. The Twenty-fifth International Offshore and Polar Engineering Conference. International Society of Offshore and Polar Engineers, 2015.

[11] Yeung RW, Kim JW. Effects of a translating load on a floating platestructural drag and plate deformation. Journal of fluids and structures 2000;14(7):993-1011. 
[12] Kashiwagi M. Transient responses of a VLFS during landing and take-off of an airplane. Journal of Marine Science and Technology 2004;9(1):14-23.

[13] Pogorelova AV. Wave Resistance of an Air-Cushion Vehicle in Unsteady Motion over an Ice Sheet, J Appl. Mech. Tech. Phys. 2008;49(1):71-79.

[14] Meylan MH, Sturova IV. Time-dependent motion of a two-dimensional floating elastic plate. J Fluids and Structures 25.3 (2009): 445-460.

[15] Pogorelova, A. V., V. M. Kozin, and A. A. Matyushina. "Stress-strain state of ice cover during aircraft takeoff and landing." Journal of Applied Mechanics and Technical Physics 56.5 (2015): 920-926.

[16] Newman J N "Channel wall effects in radiation-diffraction analysis" In Proc. 31 Intern. Workshop on Water Waves and Floating Bodies, (IWWWFB 2016) p. 117-120.

[17] Kozin VM. Resonant method of an ice cover destruction. Inventions and experiments. Natural Sciences Acad, Moscow, 2007; 354pp.

[18] http://www.dartmouth.edu/ floods/Archives/2007sum.htm

[19] http://time.com/30174/watch-china-drops-bombs-on-icebound-river-to-preventflooding

[20] Fuamba M, Bouaanani N, Marche C. Modeling of dam break wave propagation in a partially ice-covered channel. Advances in Water Resources 2007;30:2499-2510.

[21] Hinchey M, Colbourne B. Research on low and high speed hovercraft icebreaking. Canadian Journal of Civil Engineering 1995;22(1):32-42.

[22] Zhestkaya VD. Numerical solution of the problem of an ice sheet under a moving load. J App Mech Tech Phys 1999;40(4):770-75.

[23] Zhestkaya VD, Kozin VM. Ice Breaking with Air-Cushion Vessels Using a Resonant Method. Dalnauka, Vladivostok; 2003; 160pp.

[24] Brocklehurst P, Korobkin AA, Părău EI. Interaction of hydro-elastic waves with a vertical wall. J Eng Math 2010;68:215-31.

[25] Kozin VM, Zhestkaya VD, Pogorelova AV, Chizhumov SD, Dzhabailov MP, Morozov VS, Kustov AN. Applied problems of the dynamics of ice cover (in Russian). Academy of Natural Sciences, Moscow; 2008. 
[26] Daly SF. Wave propagation in ice-covered channels. J. of Hydraulic Eng. $1993 ; 119(8): 895-910$.

[27] Steffler PM, Hicks FE. Discussion of "Wave propagation in ice-covered channels". J. of Hydraulic Eng. 1994;120(12):1478-79.

[28] Korobkin AA, Khabakhpasheva TI, Papin AA. Waves propagating along a channel with ice cover. Eur J Mech B/Fluids 2014;47:166-75.

[29] Batyaev EA, Khabakhpasheva TI. Hydroelastic waves in channel with free ice cover. Fluid Dynamics 2015;6:84-101.

${ }_{580}$ [30] Wilson JT. Moving loads on floating ice sheets. Project 2432. University of Michigan Research Institute. 1958.

[31] Hosking RJ, Sneyd AD, Waugh DW. Viscoelastic response of a floating ice plate to a steadily moving load. J Fluid Mechanics 1988;196:409-430.

[32] Takizawa T. Response of a floating sea ice sheet to a moving vehicle. In Proc. Fifth International Offshore Mechanics and Arctic Engineering Symposium, Tokyo ; 1986;4: 614-621.

[33] Tabata T. Studies on visco-elastic properties of sea ice. In Arctic Sea Ice. US National Academy of Sciences \& National Research Council (Washington, DC), publication 598, pp. 139-147.

[34] Mase GE. Theory and problem of Continuum Mechanics. Schaums outline series. McGraw-Hill Book Company. 1970: 223 p.

[35] Brocklehurst P. Hydroelastic waves and their interaction with fixed structures. PhD thesis, University of East Anglia, UK; 2012.

[36] Jones F, Ryffel H, Oberg E, McCauley C. Machinerys handbook. 27th edn, Industrial Press Inc, New York; 2004.

[37] Squire V. The breakup of shore fast sea ice. Cold Reg Sci Technol 1993;21:211-18.

[38] Goodman D, Wadhams P, Squire V. The flexural response of a tabular ice island to ocean swell. Ann Glaciol 1980;1:23-7. 
[39] Squire V, Martin S. A field study of the physical properties, response to swell, and subsequent fracture of a single ice floe in the winter bering sea. Technical report 18, University of Washington; 1980. 